\title{
EXTRAPOLATION OF THE FUNCTIONAL CALCULUS OF GENERALIZED DIRAC OPERATORS AND RELATED EMBEDDING AND LITTLEWOOD-PALEY-TYPE THEOREMS. I
}

\author{
SERGEY S. AJIEV \\ (Received 6 August 2004: revised 6 June 2005) \\ Communicated by A. H. Dooley
}

\begin{abstract}
Several rather general sufficient conditions for the extrapolation of the calculus of generalized Dirac operators from $L_{2}$ to $L_{p}$ are established. As consequences. we obtain some embedding theorems. quadratic estimates and Littlewood-Paley theorems in terms of this calculus in Lebesgue spaces. Some further generalizations. utilised in Part II devoted to applications. which include the Kato square root model. are discussed. We use resolvent approach and show the irrelevance of the semigroup one. Auxiliary results include a high order counterpart of the Hilbert identity, the derivation of new forms of 'off-diagonal' estimates. and the study of the structure of the model in Lebesgue spaces and its interpolation properties. In particular. some coercivity conditions for forms in Banach spaces are used as a substitution of the ellipticity ones. Attention is devoted to the relations between the properties of perturbed and unperturbed generalized Dirac operators. We do not use any stability results.

2000 Mathematics subject classification: primary 46E15, 46E.30, 47A60, 47A65: secondary 47A05, 47A55, 46B20. 46C99. 42C99.

Keywords and phrases: functional calculus. generalized embedding. Littlewood-Paley theorem. extrapolation of singular operators, generalized Dirac operator. Hilbert identity. off-diagonal estimates. Kato square root model.
\end{abstract}

\section{Introduction}

The recent complete solution of the long standing square root problem of Kato for elliptic operators and systems by Auscher, Hofmann, Lacey, McIntosh and Tchamitchian in $[7,8,20]$ was preceded by works of McIntosh [23] and Coifman, McIntosh, Meyer [13], as well as a book due to Auscher and Tchamitchian [9] devoted to the boundedness of the square roots of elliptic operators on $L_{2}$.

(c) 2007 Australian Mathematical Society $1446-7887 / 07 \$ A 2.00+0.00$ 
Later Auscher [5] together with Coulhon, Duong and Hofmann [6] extended this result for elliptic operators to a range of Lebesgue spaces employing ideas originating from the recent results of Blunck and Kunstman [12] and Martell [22] with Hofmann [19]. The former source was, in turn, improving results of Duong, McIntosh [16], Duong, Robinson [17] and Hebisch [18]. Other generalizations of extrapolation results [12], intersecting partly with [5], were also obtained in $[2,3]$ using a different approach.

The above mentioned developments are known to be partly based and intimately related to the theory of $H^{\infty}$-calculus developed by McIntosh [24], Albrecht, Duong, McIntosh [4], and Cowling, Doust, McIntosh, Yagi [14] in the abstract settings of Hilbert and Banach spaces correspondingly.

Contemporary development of the $L_{2}$-theory is represented by the work [10] due to Axelsson, Keith and McIntosh, where the model of generalized Dirac operator, which is more general than one associated with the Kato Square root problem, was investigated. They established the existence of $H^{\infty}$-functional calculus of a perturbed Dirac operator on $L_{2}\left(\mathbb{R}^{n}\right)$ and related quadratic estimates, including a possible approach to the solution of the Square root problem of Kato as a particular case.

The results of this paper are extensions and generalizations of [2, Chapter IV], where the case of 'lower' extrapolation was considered in the case of an injective $\Pi_{B}$.

The majority of the article is, roughly speaking, related to [10] in a similar way as $[5,6,19]$ is related to the recent solution of the Kato problem. More precisely, we find several groups of the conditions are sufficient to extrapolate the existence of $H^{\infty}$ functional calculus in $L_{2}$, of the generalized Dirac operator in the form considered in [10], to its existence in $L_{p}$ and to establish quadratic estimates and LittlewoodPaley theorems in terms of this calculus for a reasonably wide range of $p$ contained in $(1, \infty)$. To extend the range of the possible applications considered in Part II, a sufficiently high level of generality is maintained, and some approaches to further extensions and generalizations are discussed in Section 6. Other results of Section 3 are generalized embedding theorems for generalized Dirac operators written in the form of the boundedness of its powers from Besov and Lizorkin-Triebel spaces into Lebesgue (Lorentz) ones.

For this purpose, we use the resolvent approach in the derivation of new generalized off-diagonal estimates without any stability considerations. Some of these conditions are given a weighted form permitting us to deal with subspaces of $L_{p}$. The usual off-diagonal conditions cannot be used due to their local nature. As collateral results, our approach delivers the extensions of the Hilbert resolvent identity to the case of differences of a high order (in Section 4).

To demonstrate the irrelevance of the traditionally more popular semigroup approach in our settings, in Section 6, we develop a one-dimensional model case suggested by McIntosh. 
The transference of the model of the generalized Dirac operator, introduced in [10] in the Hilbert space $L_{2}$, to $L_{p}$-spaces is achieved with the idea of the form coercivity originating from [21]. This is a weaker hypothesis than the ellipticity assumption used in [10] to establish $L_{2}$-calculus. Despite the fact that our form coercivity condition for an operator $B: L_{p} \rightarrow L_{p}$ is stronger than that considered in [25] (which is equivalent to the boundedness of $B^{-1}$ ), it is still weaker for $p=2$ than the restriction $|(f, B f)| \geq C\left\|f \mid L_{2}\right\|^{2}$.

\section{Definitions and designations}

In this section, we define two types of off-diagonal families of functions with respect to some operators and the main assumptions on the operators employed, such as properties: General $\mathbf{G}(p)$, Coercivity $\mathbf{C}$, Idempotence I, Projectivity $\mathbf{P}(q)$, Weak Projectivity WP, Local Closure LC, Bounded Coercivity $\mathbf{B C}(p)$, and some of their adjoint properties. Some of them are trivially valid for particular applications, but required to maintain the level of generality.

The next designations are used extensively:

$$
\begin{aligned}
R(z) & :=(1-z)^{-1} ; \quad \gamma_{\nu}^{ \pm}:=\left\{t e^{ \pm i v}\right\}_{t>0}, \quad \gamma_{\nu}=\gamma_{\nu}^{+} \cup \gamma_{\nu}^{-}, \quad \gamma_{v}^{d}=-\gamma_{v} \cup \gamma_{\nu}, \\
S_{\mu} & :=\{z \in \mathbb{C} \backslash\{0\}:|\arg z|<\mu\} \quad \text { for } \mu \in[0, \pi / 2), \quad S_{\mu}^{d}:=-S_{\mu} \cup S_{\mu},
\end{aligned}
$$

and $\bar{S}_{\mu}^{d}$ is the closure of $S_{\mu}^{d}$ without $0, \bar{S}_{0}^{d}:=\mathbb{R} \backslash\{0\}$. The symbol $H_{\infty}\left(S_{\mu}^{d}\right)$ denotes the space of all bounded and holomorphic functions on $S_{\mu}^{d}$; and $\Xi\left(S_{\mu}^{d}\right)$ is the subset of the functions $f \in H_{\infty}\left(S_{\mu}^{d}\right)$ such that $f(z)=\mathrm{O}\left(|z|^{-\alpha}\right)$ for $|z| \rightarrow \infty$ and some $\alpha>0$; By $\Psi\left(S_{\mu}^{d}\right)$ we denote the subset of functions $f \in H_{\infty}\left(S_{\mu}^{d}\right)$ such that $|f(z)| \leq$ $C\left|z /\left(1+z^{2}\right)\right|^{\alpha}$ for any $z \in S_{\mu}^{d}$ and some $\alpha>0$. Let $Q\left(S_{\mu}^{d}\right)$ be the subset of functions $f \in H_{\infty}\left(S_{\mu}^{d}\right)$ such that

$$
Q\left(S_{\mu}^{d}\right):=\left\{f \in H_{\infty}\left(S_{\mu}^{d}\right): \sup _{z \in S_{\mu}^{d}} \int_{0}^{\infty}|f(z t)| d t / t<\infty\right\} .
$$

Let $F\left(S_{\mu}^{d}\right)$ be the class of all holomorphic functions $f$ on $S_{\mu}^{d}$ satisfying, for some $\alpha \in \mathbb{R},|f(z)| \leq C\left(|z|^{\alpha}+|z|^{-\alpha}\right)$. By means of $\bar{H}_{\infty}\left(S_{\mu}^{d} \cup\{0\}\right)$, we denote the subspace of $L_{\infty}\left(S_{\mu}^{d} \cup\{0\}\right)$ containing all everywhere-finite functions holomorphic on $S_{\mu}^{d}$,

$$
\bar{H}_{\infty}^{0}\left(S_{\mu}^{d} \cup\{0\}\right)=\left\{f \in \bar{H}_{\infty}\left(S_{\mu}^{d} \cup\{0\}\right): f(0)=0\right\} .
$$

We shall use the term linear (sub)space if the corresponding (sub)space is not required to be complete (closed). Because, along with $L_{p}$, other Banach spaces with a few parameters will be dealt with, we adopt some traditional notation from 
the theory of function spaces. For a Banach space $A$ and some $x \in A$, let $\|x \mid A\|$ denote the norm of $x$ in $A$. For example, if $B$ is a Banach space, and $G$ and $f$ is a measurable subset of $\mathbb{R}^{n}$ and some Bochner-measurable function defined on it, then $\left\|f \mid L_{p}(G, B)\right\|$ is the norm of the function $f$ in the vector-valued Lebesgue space $L_{p}(G, B)$. When there is no ambiguity concerning $G$ and $B$, sometimes we use the designation $\|f\|_{p}:=\left\|f \mid L_{p}(G, B)\right\|$.

For $p \in(0, \infty)$, let $M_{p} f:=\left(M|f|^{p}\right)^{1 / p}$, where $M f$ is the Hardy-Littlewood maximal function.

For $N \in \mathbb{N}, p, t>0$, and $w, x \in \mathbb{R}^{n}$, let $\rho_{t, u}(x):=\min (1, t /|x-w|)$ and $L_{p, \rho_{i .1 .}^{N}}$ be the weighted Lebesgue space of the measurable functions $f$ with finite norm $\left\|f \rho_{t, \ldots}^{N}(\cdot) \mid L_{p}\left(\mathbb{R}^{n}\right)\right\|$.

Let $w_{p}^{1}=w_{p}^{1}\left(\mathbb{R}^{n}, \mathbb{R}^{m}\right)$ be a seminormed (homogeneous) Sobolev space of functions $f$ with the finite $\left\|f\left|w_{p}^{1}\|=\| \nabla f\right| L_{p}\left(\mathbb{R}^{n}, \mathbb{C}^{m n}\right)\right\|$. We say that a pair $(p, q) \in \mathbb{R}_{+}^{2}$ is in the Sobolev relation $\mathscr{S}_{1} \subset \mathbb{R}_{+}^{2}$ if $W_{p}^{1} \subset L_{q}$, where $W_{p}^{1}$ is defined by

$$
\left\|f\left|W_{p}^{1}\|=\| f\right| L_{p}\right\|+\left\|f \mid w_{p}^{1}\right\| .
$$

For $p, q \in[1, \infty], s>0$, the symbols $b_{p . q}^{s}$ and $l_{p . q}^{s}$ denote, correspondingly, the seminormed (homogeneous) spaces of Besov and Lizorkin-Triebel type of the functions $f$ defined on $\mathbb{R}^{n}$ and possessing, respectively, the finite norms:

$$
\begin{aligned}
& \left\|f \mid b_{p, q}^{s}\right\|:=\left(\int_{0}^{\infty}\left(t^{-s}\left\|\delta^{m}(t, \cdot, f) \mid L_{p}\left(\mathbb{R}^{n}\right)\right\|\right)^{q} \frac{d t}{t}\right)^{1 / q} \text { and } \\
& \left\|f\left|l_{p, q}^{s}\|:=\|\left(\int_{0}^{\infty}\left(t^{-s} \delta^{m}(t, \cdot, f)\right)^{q} \frac{d t}{t}\right)^{1 / q}\right| L_{p}\left(\mathbb{R}^{n}\right)\right\|,
\end{aligned}
$$

where the integer $m>s$ and

$$
\delta^{m}(t, x, f):=2^{-n} \int_{Q_{0}}\left|\Delta_{t h}^{m} f(x)\right| d h \quad \text { for } Q_{0}:=[-1,1]^{n} .
$$

DEFINITION 2.1. For a measurable function $f$, let $\sigma(t, f)=|\{x:|f(x)|>t\}|$ and $f^{*}(t):=\inf \{\tau: \sigma(\tau, f) \leq t\}$ for some $t>0$. Following [1], a measurable $\mathbb{C}^{m}$-valued function $f$ on $\mathbb{R}^{n}$ is Chebyshev-regular if $\lim _{t \rightarrow \infty}\left(\left\|f \mid \mathbb{C}^{m}\right\|\right)^{*}(t)=0$. For an arbitrary function space $Y=Y\left(\mathbb{R}^{n}, \mathbb{C}^{m}\right)$, we designate its subspace of all Chebyshev-regular functions endowed with the inherited (semi)norm by $\dot{Y}=\dot{Y}\left(\mathbb{R}^{n}, \mathbb{C}^{m}\right)$.

Let $X$ and $Y$ be Banach spaces and $A$ be an operator with $E \cup D(A) \subset X$ and $R(A) \subset Y$. Then the symbol $A_{\mid E}$ denotes the restriction of $A$ to $E$, that is $D\left(A_{\mid E}\right)=E \cap D(A)$ and $A_{\mid E} x=A x$ for every $x \in E \cap D(A)$. For subspaces $M$ and $N$ of a Banach space $X$ with $M \cap N=0, M \oplus N$ denotes its topological sum. 
[5] Extrapolation of functional calculus, embedding theorems and Littlewood-Paley inequalities 301

For Banach spaces $X$ and $Y$, let $\mathscr{C}(X, Y)$ be the space of all closed linear operators with domains in $X$ and ranges in $Y ; \mathscr{C}(X):=\mathscr{C}(X, X)$. For operators $A: D(A) \rightarrow Y$ and $B: D(B) \rightarrow Y$, one writes $A \subset B$ if $D(A) \subset D(B)$ and $A=B_{\mid D(A)}$. For a Banach space $X$ and a linear space $E$, the set $X_{E}:=X \cap E$ is a well defined linear subspace of $X$, we denote the normed space $X_{E}$ with the norm $\|\cdot \mid X\|$ by $X \cap E$.

For any $p, q \in(0, \infty]$ and an operator $A$ from $L_{p}$ into $L_{q}$, let $D_{p . q}(A), N_{p . q}(A)$ and $R_{p . q}(A)$ be the domain, kernel and range of $A$ respectively, and $D_{p}(A):=D_{p . p}(A)$, $N_{p}(A):=N_{p, p}(A)$ and $R_{p}(A):=R_{p, p}(A)$. We write $N_{p, \rho_{i, k}^{N}}(A)$ and $N_{p, \rho_{t, w}^{N}}(A)$ if the corresponding Lebesgue space $L_{p}\left(\mathbb{R}^{n}\right)$ is substituted for its weighted counterpart $L_{p . \rho_{t, k}^{N}}\left(\mathbb{R}^{n}\right)$.

For Banach spaces $X$ and $Y$ and their linear subspaces $D_{X} \subset X$ and $D_{Y} \subset Y$, a semi-linear form $b: D_{X} \times D_{Y} \rightarrow \mathbb{C}$ is understood to be bounded if

$$
|b(x, y)| \leq C\|x|X\|\cdot\| y| Y\|
$$

for any $(x, y) \in D_{X} \times D_{Y}$. The form $b$ is also understood to be coercive if there is a mapping $\phi=\phi_{b}: D_{Y} \rightarrow D_{X}$ satisfying

$$
\|\phi(y)|X\|\leq C\| y| Y\| \quad \text { and } \quad\left\|y\left|Y \|^{2} \leq C\right| b(\phi(y), y) \mid \text { for all } y \in D_{Y} .\right.
$$

Let $b^{*}(y, x):=\overline{b(x, y)}$ be the corresponding adjoint form.

Dealing with functions of a closed operator $A$, we assume or describe the presence of a mapping $\mathscr{F}:=\mathscr{F}_{A}: f \mapsto f(A)$ from a set of functions $D(\mathscr{F})$ into $\mathscr{C}(X, Y)$, where $X$ and $Y$ are Banach spaces. The mapping $\mathscr{F}$ satisfies the conditions:

(1) $\mathscr{F}(0)=0$ if $0 \in D(\mathscr{F})$;

(2) $\mathscr{F}(1)=I$ if $1 \in D(\mathscr{F})$;

(3) $\mathscr{F}(z)=A$ if $z \in D(\mathscr{F})$;

(4) $\mathscr{F}(\alpha f+\beta g)=\alpha \mathscr{F}(f)+\beta \mathscr{F}(g)$ if $\alpha, \beta \in \mathbb{C}$ and $f, g, \alpha f+\beta g \in D(\mathscr{F})$;

(5) $\mathscr{F}(f g)=\mathscr{F}(f) \mathscr{F}(g)$ if $f, g, f g \in D(\mathscr{F})$.

REMARK 1. (a) In most cases, $D(\mathscr{F})$ is a subset of a normed space $X$ with $\delta_{0}: f \mapsto f(0)$ being in $X^{*}$ and $R(\mathscr{F}) \subset \mathscr{L}(Y, Z)$, where $Y, Z$ are Banach spaces and $Y \subset Z$ (that is $I \in \mathscr{L}(Y, Z)$ ). Therefore, to establish that the mapping $\mathscr{F}$ is well defined on $D(\mathscr{F})$, we consider its action on the set $D^{0}(\mathscr{F})=D(\mathscr{F}) \cap N\left(\delta_{0}\right)$ only. Indeed, one has

$$
\mathscr{F} f=\mathscr{F}\left(f-\delta_{0}(f)\right)+\delta_{0}(f) \mathscr{F} 1 .
$$

Therefore,

$$
\mathscr{F}_{\mid D^{0}(\mathscr{F})} \in \mathscr{L}\left(X \cap D^{0}(\mathscr{F}), \mathscr{L}(Y, Z)\right) \quad \Rightarrow \quad \mathscr{F} \in \mathscr{L}(X \cap D(\mathscr{F}), \mathscr{L}(Y, Z)),
$$

because $\delta_{0} \in X^{*}$ and $\mathscr{F} 1=I \in \mathscr{L}(Y, Z)$. 
(b) This approach appears to be especially useful in the settings where $N(A)$ is complemented in $Y, Y=N(A) \oplus Y_{1}$, and there is another mapping $\mathscr{F}_{1}$ with $R\left(\mathscr{F}_{1}\right) \subset \mathscr{L}\left(Y_{1}, Z\right)$. We intend to construct a functional calculus on $Y$. Namely, it coincides, in this case, with the degenerate spectral projector approach from [10], that is, $\mathscr{F}: f(z) \mapsto f(0) \mathrm{P}_{0}+f\left(A_{\mid Y_{1}}\right)\left(I-\mathrm{P}_{0}\right)$, where $\mathrm{P}_{0}$ is the bounded projector in $X$ with $R\left(\mathrm{P}_{0}\right)=N(A)$ and $N\left(\mathrm{P}_{0}\right)=Y_{1}$ and $\mathscr{F}_{A_{Y Y}}$ is defined as in the injective case $(N(A)=\{0\})$.

For $\omega \in[0, \pi / 2)$ and Banach spaces $X$ and $Y$, we assume that an operator $A \in \mathscr{C}(X, Y)$ is of type $\omega$ if, for any $z \in \mathbb{C} \backslash \bar{S}_{\omega}^{\prime \prime}$, one has $R(z A) \in \mathscr{L}(X, Y)$ and

$$
\|R(z A) \mid \mathscr{L}(X, Y)\| \leq C(\arg z)
$$

where the resolvent $R(z A):=(I-z A)^{-1}$. Note that (see Definition 2.10)

$$
\mathbf{F}\left(A, X, Y, S_{\omega}^{d}\right) \quad \Longrightarrow \quad A \text { is of type } \omega .
$$

Let us define 'off-diagonal' by summability and 'double off-diagonal', or 'offdiagonal' by space and summability, classes of families of functions.

DEFINITION 2.2. Let $p, q \in[1, \infty], \mathscr{F}=\mathscr{F}_{A}, R \subset \mathbb{C}$, and $X \subset L_{p}$ is a linear subspace. We say that a function family $\left\{f_{z}\right\}_{: \in R} \subset D(\mathscr{F})$ is in the class $\bar{D}(A, R, p, q, X)$ for some $\mu_{1} \in[0, \pi / 2)$ and an operator $A$ and write

$$
\left\{f_{z}\right\}_{z \in R} \in \bar{D}(A, R, p, q, X)
$$

if, for any $\phi \in X$, one has

$$
\begin{gathered}
\left\|f_{z}(A) \phi\left|L_{q}\left(\mathbb{R}^{n}\right)\left\|\leq C|z|^{n / q-n / p}\right\| \phi\right| L_{p}\left(\mathbb{R}^{n}\right)\right\| \text { for all } z \in S_{\mu_{1}} \\
\bar{D}\left(A, S_{\mu_{1}}, p, q\right):=\bar{D}\left(A, S_{\mu_{1}}, p, q, L_{p}\right)
\end{gathered}
$$

We say that a function family $\left\{f_{z}\right\}_{: \in R} \subset D(\mathscr{F})$ is in the class $\bar{D}(A, R, p, q, N, X)$ for some $p, q \in[1, \infty], N>0$, an operator $A$ and a linear subspace $X \subset L_{l}$, and write

$$
\left\{f_{z}\right\}_{z \in R} \in \bar{D}(A, R, p, q, N, X)
$$

if for any $\phi \in X$, one has

$$
\left\|f_{z}(A) \phi\left|L_{q}\left(Q_{|z|}(w)\right)\left\|\leq C|z|^{n / q-n / p}\right\| \phi\right| L_{p \cdot \rho_{i=1.4}^{*}}\left(\mathbb{R}^{n}\right)\right\|
$$

for all $z \in S_{\mu_{1}}, w \in \mathbb{R}^{n}$ and $\phi \in X$. 
[7] Extrapolation of functional calculus, embedding theorems and Littlewood-Paley inequalities 303

Therefore, one has $\bar{D}\left(A, S_{\mu_{1}}, p, q, X\right)=\bar{D}\left(A, S_{\mu_{1}}, p, q, X, 0\right)$.

We make the following geometric observations because they help avoid introducing unnecessary restrictions.

REMARK 2. Consider

(a) $\left\{f_{z}\right\}_{z \in S_{\mu_{1}}} \in \bar{D}\left(A, S_{\mu_{1}}, p, q, N, X\right)$;

(b) for some $N_{1} \in \mathbb{N}$ and any $w_{0}, w_{1} \in \mathbb{R}^{n}$ and $\phi \in X$ with supp $\phi \subset Q_{|=|}\left(w_{0}\right)$, one has

$$
\left\|f_{z}(A) \phi\left|L_{q}\left(Q_{t}\left(w_{1}\right)\right)\left\|\leq C|z|^{n / 4-n / p}\left(\rho_{|z| \cdot w_{0}}\left(w_{1}\right)\right)^{N_{1}}\right\| \phi\right| L_{p}\right\| \quad \text { for all } z \in S_{\mu_{1}}
$$

(c) for some $N_{2} \in \mathbb{N}$ and any $w \in \mathbb{R}^{n}$ and $\phi \in X$ with $\operatorname{supp} \phi \subset Q_{\mid=1}(w)$, one has

$$
\left\|f_{z}(A) \phi\left|L_{q, \rho_{i|l| w}^{-N_{2}}}\left(\mathbb{R}^{n}\right)\left\|\leq C|z|^{n / q-n / p}\right\| \phi\right| L_{p}\left(Q_{|=|}(w)\right)\right\| \quad \text { for all } z \in S_{\mu_{1}} .
$$

Using the notation of the previous definition, one has the implications:

(1) (a) implies (b) implies (c) for $N \geq N_{1} \geq N_{2}+n$;

(2) (a) if and only if (b) if and only if (c) for $X=L_{p}$ and $N, N_{1}, N_{2}$ chosen appropriately for each particular implication.

Definition 2.3. For $p, q \in(0, \infty], p \leq q$ and a linear subspace $X \subset L_{q}$, let $A: D_{q . p} \rightarrow L_{p}$ be an injective operator. Then we say that $\mathbf{S}(A, p, q, X)$, or the Sobolev property, holds if

$$
\left\|f\left|L_{q}\|\leq C(A)\| A f\right| L_{p}\right\| \text { for all } f \in X \subset D_{q, p}(A) .
$$

We designate $\Delta^{k} f(z):=\sum_{l=0}^{k}(-1)^{l}\left(\begin{array}{l}k \\ l\end{array}\right) f(l z), k \in \mathbb{N}$ for any (operator-valued) function $f(z)$.

We shall often deal with operators $\Gamma, \Gamma^{*} \in \mathscr{C}\left(L_{p}\right)$ where $D_{p}(\Gamma)$ and $D_{p}\left(\Gamma^{*}\right)$ are dense in $L_{p}$ for some $p \in(1, \infty)$ and their combinations $\Pi=\Pi_{I}=\Gamma+\Gamma^{*}$, $\Gamma_{B^{*}}:=B_{2}^{*} \Gamma B_{1}^{*}, \Gamma_{B}^{*}:=B_{1} \Gamma^{*} B_{2}, \Pi_{B}:=\Gamma+\Gamma_{B}^{*}$, and $\Pi_{B^{*}}^{*}:=\Gamma^{*}+\Gamma_{B^{*}}$ with operators $B_{1}, B_{2} \in \mathscr{L}\left(L_{p}\right)$ or $B_{1}^{*}, B_{2}^{*} \in \mathscr{L}\left(L_{p}\right)$ imposing some of the following conditions and assuming

$$
\begin{aligned}
& D_{p}(\Gamma)=D_{p}\left(\Gamma^{2}\right)=D_{p}\left(\Gamma B_{1}^{*} B_{2}^{*} \Gamma\right) \\
& \text { and } D_{p}\left(\Gamma^{*}\right)=D_{p}\left(\Gamma^{* 2}\right)=D_{p}\left(\Gamma^{*} B_{2} B_{1} \Gamma^{*}\right), \\
& \text { and } \Gamma^{2} \subset 0, \quad \Gamma^{* 2} \subset 0 \text { and } \Gamma B_{1}^{*} B_{2}^{*} \Gamma \subset 0, \quad \Gamma^{*} B_{2} B_{1} \Gamma^{*} \subset 0 .
\end{aligned}
$$

When the inclusions of the previous line take place, we say that $\mathbf{I}$, or the idempotence condition, holds. 
DEFINITION 2.4. Let $p \in(0, \infty]$. We say that $\mathbf{G}(p)$, or the general condition holds if

$$
\begin{gathered}
B_{1}, B_{2} \in \mathscr{L}\left(L_{p}\right), \text { there exists bounded }\left(B_{1 \mid \overline{R_{p}\left(\Gamma^{*}\right)}}\right)^{-1},\left(B_{2 \mid \overline{R_{p}(\Gamma)}}\right)^{-1} \text {, and } \\
D_{p}\left(\Pi_{B}\right)=D_{p}(\Gamma) \cap D_{p}\left(\Gamma_{B}^{*}\right) \text { is dense in } L_{p},
\end{gathered}
$$

where the closures of $R_{p}(\Gamma)$ and $R_{p}\left(\Gamma^{*}\right)$ in $L_{p}$ have inherited the $L_{p}$-topology. We say that $\mathbf{G}^{*}(p)$, or the adjoint general condition, holds if

$$
\begin{gathered}
B_{1}^{*}, B_{2}^{*} \in \mathscr{L}\left(L_{p^{\prime}}\right), \text { there exists bounded }\left(B_{1 \mid \overline{R_{p^{\prime}}}\left(\Gamma^{*}\right)}^{*}\right)^{-1},\left(B_{2 \mid \overline{R_{p^{\prime}}(\Gamma)}}^{*}\right)^{-1} \text {, and } \\
D_{p^{\prime}}\left(\Pi_{B^{*}}^{*}\right)=D_{p^{\prime}}\left(\Gamma^{*}\right) \cap D_{p^{\prime}}\left(\Gamma_{B^{*}}\right) \text { is dense in } L_{p^{\prime}},
\end{gathered}
$$

where the closures of $R_{p^{\prime}}(\Gamma)$ and $R_{p^{\prime}}\left(\Gamma^{*}\right)$ in $L_{p^{\prime}}$ have inherited the $L_{p^{\prime}}$-topology.

If $\mathbf{G}(p)$ or $\mathbf{G}^{*}(p)$ is true with the density requirement omitted, we say that the restricted general condition holds.

We say that $\mathbf{C}$, or the coercivity condition, holds if

$$
\begin{gathered}
\left\|\Pi f\left|L_{2}\|C \geq\| f\right| w_{2}^{1}\right\| \quad \text { for all } f \in D_{2}(\Pi) \cap \overline{R_{2}(\Pi)} \quad \text { and } \\
\bigcap_{q_{s} \leq p \leq q_{s}^{\prime}} D_{p}(\Pi) \text { is dense in } L_{p}
\end{gathered}
$$

for every $p \in\left[q_{s}, q_{s}^{\prime}\right]$ and $\left(q_{s}, 2\right) \in \mathscr{S}_{1}$.

REMARK 3. (a) One should point out that the proofs of the results in Sections 3 and 4 show that we can use the appropriate Sobolev property (of the form $\mathbf{S}(\Pi, 2, q, X))$ instead of the Coercivity property $(\mathbf{C})$.

(b) Under the conditions $\mathbf{G}(p)$ and $\mathbf{P}(p)\left(\mathbf{G}^{*}(p)\right.$ and $\left.\mathbf{P}^{*}(p)\right)$, the operators $\Gamma_{B}^{*}$ and $\Pi_{B}\left(\Gamma_{B^{*}}\right.$ and $\left.\Pi_{B^{*}}^{*}\right)$ are closed if $\Gamma$ and $\Gamma^{*}$ are closed, or $\Gamma$ is closed and $\mathbf{D}(p)$ holds.

(c) The smoothness of the order $1\left(w_{2}^{1}\right.$ and $\left.\mathscr{S}_{1}\right)$ is taken for the sake of simplicity.

Definition 2.5. For $p \in[1, \infty], i=1,2$ and $A_{1}=\Gamma^{*}, A_{2}=\Gamma$, let the forms $b_{i}: L_{p^{\prime}} \times L_{p} \rightarrow \mathbb{C}$ be defined by $b_{i}(f, g):=\int_{\mathbb{g}^{n}} \bar{f} B_{i} g$. Then we say that $\mathbf{B C}(p)$, or the bounded coercivity condition, holds if, for $i=1,2$ :

$b_{i}$ is bounded on $L_{p^{\prime}} \times L_{p}$ and coercive on $R_{p^{\prime}}\left(A_{i}\right) \times R_{p}\left(A_{i}\right)$.

We also say that $\mathbf{B C}^{*}(p)$, or the adjoint bounded coercivity condition, holds if, for $i=1,2$ :

$b_{i}^{*}$ is bounded on $L_{p} \times L_{p^{\prime}}$ and coercive on $R_{p}\left(A_{i}\right) \times R_{p^{\prime}}\left(A_{i}\right)$. 
[9] Extrapolation of functional calculus, embedding theorems and Littlewood-Paley inequalities 305

DEFINITION 2.6. We say that $\mathbf{D}(p)$, or the duality condition, holds if

$$
\begin{gathered}
\int_{\mathbb{R}^{n}} \bar{f} \Gamma g=\int_{\mathbb{R}^{n}} g \overline{\Gamma^{*} f} \text { and } \int_{\mathbb{R}^{n}} \bar{f} B_{i} g=\int_{\mathbb{R}^{n}} g \overline{B_{i}^{*} f}, i=1,2 \\
\quad \text { for }(f, g) \in D_{p^{\prime}}\left(\Gamma^{*}\right) \times D_{p}(\Gamma) \cup D_{p}\left(\Gamma^{*}\right) \times D_{p^{\prime}}(\Gamma)
\end{gathered}
$$

DEFINITION 2.7. We say that $\mathbf{P}(p)$, or the projectivity condition, holds if

$$
L_{p}=N_{p}\left(\Pi_{B}\right) \oplus \overline{R_{p}\left(\Pi_{B}\right)}, \quad \overline{R_{p}\left(\Pi_{B}\right)}=\overline{R_{p}(\Gamma)} \oplus \overline{R_{p}\left(\Gamma_{B}^{*}\right)}
$$

We also say that $\mathbf{P}^{*}(p)$, or the adjoint projectivity condition, holds if

$$
L_{p^{\prime}}=N_{p^{\prime}}\left(\Pi_{B^{*}}^{*}\right) \oplus \overline{R_{p^{\prime}}\left(\Pi_{B^{*}}^{*}\right)}, \quad \overline{R_{p^{\prime}}\left(\Pi_{B^{*}}^{*}\right)}=\overline{R_{p^{\prime}}\left(\Gamma^{*}\right)} \oplus \overline{R_{p^{\prime}}\left(\Gamma_{B^{*}}\right)} .
$$

We designate the corresponding projections on $N_{p}\left(\Pi_{B}\right), \overline{R_{p}(\Gamma)}, \overline{R_{p}\left(\Gamma_{B}^{*}\right)}, N_{p^{\prime}}\left(\Pi_{B^{*}}^{*}\right)$, $\overline{R_{p^{\prime}}\left(\Gamma^{*}\right)}$ and $\overline{R_{p^{\prime}}\left(\Gamma_{B^{*}}\right)}$ by means of $P_{0}=P_{0 . p}, P_{1}=P_{1, p}, P_{2}=P_{2 . p}, P_{0}^{*}=P_{0 . p}^{*}$, $P_{1}^{*}=P_{1, p}^{*}$ and $P_{2}^{*}=P_{2, p}^{*}$ respectively.

COROLlaRY 2.8. (a) If $\mathbf{P}(p)$ holds, then $\Gamma A, A \Gamma_{B}^{*} \subset P_{1 . p}, \Gamma_{B}^{*} A, A \Gamma \subset P_{2, p}$ and $\Gamma A=A \Gamma_{B}^{*}$ on $D_{p}\left(\Gamma_{B}^{*}\right) \cap R_{p}\left(\Pi_{B}\right)$ and $\Gamma_{B}^{*} A=A \Gamma$ on $D_{p}(\Gamma) \cap R_{p}\left(\Pi_{B}\right)$, where $A:=\left(\Pi_{B \mid \overline{R_{p}\left(\Pi_{B}\right)}}\right)^{-1}$;

(b) If $\mathbf{P}^{*}(p)$ holds, then $\Gamma^{*} A_{*}, A_{*} \Gamma_{B^{*}} \subset P_{1, p^{\prime}}^{*}, \Gamma_{B^{*}} A_{*}, A_{*} \Gamma^{*} \subset P_{2, p^{\prime}}^{*}$ and $\Gamma^{*} A_{*}=$ $A_{*} \Gamma_{B^{*}}$ on $D_{p^{\prime}}\left(\Gamma_{B^{*}}\right) \cap R_{p^{\prime}}\left(\Pi_{B^{*}}^{*}\right)$ and $\Gamma_{B^{*}} A_{*}=A_{*} \Gamma^{*}$ on $D_{p^{\prime}}\left(\Gamma^{*}\right) \cap R_{p^{\prime}}\left(\Pi_{B^{*}}^{*}\right)$, where $A_{*}:=\left(\Pi_{B^{*} \mid \overline{R_{\rho^{\prime}}}\left(\Pi_{B^{*}}^{*}\right)}\right)^{-1}$.

DEFINITION 2.9. We say that WP, or the weak projectivity condition, holds if $\Pi_{\mid \overline{R_{p}(\Gamma)}}$ and $\Pi_{\mid \overline{R_{p}\left(\Gamma^{*}\right)}}$ are injective for every $p \in\left[q_{s}, q_{s}^{\prime}\right]$ and $\left(q_{s}, 2\right) \in \mathscr{S}_{1}$.

DEFINITION 2.10. For an open $\Omega \subset \mathbb{C}$ and Banach spaces $X$ and $Y$, the designation $\mathbf{F}(A, X, Y, \Omega)$ means that an operator $A \in \mathscr{C}(X, Y)$ possesses the bounded $\bar{H}_{\infty}(\Omega)$ functional calculus on $L_{p}$, that is, the homomorphism $\mathscr{F}$ described above satisfies

$$
D(\mathscr{F})=\bar{H}_{\infty}(\Omega) \quad \text { and } \quad \mathscr{F} \in \mathscr{L}\left(\bar{H}_{\infty}(\Omega), \mathscr{L}(X, Y)\right) \text {. }
$$

To take advantage of homogeneity arguments, we use the stretching operators $\sigma_{t}: f \mapsto f(\cdot / t), t>0$. For an operator $A$ with $\overline{\sigma_{t}(R(A))}=\overline{R(A)}$ for every $t>0$, by means of $A_{t}$, we denote the composition $\sigma_{t}^{-1} A \sigma_{t}$.

DEFINITION 2.11. Given a condition $\mathbf{Z}$, we say that $\mathbf{H}[\mathbf{Z}]$, or the homogeneity property with respect to $\mathbf{Z}$, holds if the condition $\mathbf{Z}$ is satisfied uniformly in $t>0$ by the operators $\Gamma_{t}, \Gamma_{t}^{*}, B_{1 t}, B_{2 t}$ substituting the operators $\Gamma, \Gamma^{*}, \Pi_{B}, \Pi_{B^{*}}^{*}, B_{1}, B_{2}$ correspondingly, and

$$
\overline{R\left(A_{t}\right)}=\overline{R(A)} \text { for all } t>0 \text { and } A \in\left\{\Gamma, \Gamma^{*}, \Pi_{B}, \Pi_{B^{*}}^{*}, B_{1}, B_{2}\right\} .
$$


DEFINITION 2.12. We say that LC, or the local closure condition, holds if:

$$
L_{p} \text {-closure of } L_{p} \cap \overline{R_{2}(\Pi)} \text { (closure in } L_{2} \text { )-contains } R_{p}(\Pi)
$$

for any $p \in\left[p_{s}, 2\right),\left(p_{s}, 2\right) \in \mathscr{S}_{1}$.

DEFINITION 2.13. Let $p \in[1, \infty]$. We say that $\mathbf{K}(p)$, or the $K$-condition, holds if the closure of $D_{p}(\Gamma) \cap D_{2}(\Gamma) L_{p}+L_{2}$ contains $\overline{R_{p}\left(\Pi_{B}\right)} \cap \overline{R_{2}\left(\Pi_{B}\right)}$, and

$$
P_{0 . p \mid N_{z}\left(\Pi_{B}\right)}=P_{0.2 \mid N_{p}\left(\Pi_{B}\right)} \text {. }
$$

We also say that $\mathbf{K}^{*}(p)$, or the adjoint $K$-condition, holds if the closure of $D_{p^{\prime}}\left(\Gamma^{*}\right) \cap$ $R_{2}\left(\Gamma^{*}\right) L_{p^{\prime}}+L_{2}$ contains $\overline{R_{p^{\prime}}\left(\Pi_{B}\right)} \cap \overline{R_{2}\left(\Pi_{B}\right)}$, and

$$
P_{0 . p^{\prime} \mid N_{2}\left(\Pi_{B^{*}}^{*}\right)}=P_{0.2 \mid N_{r^{\prime}}\left(\Pi_{B^{*}}^{*}\right)} .
$$

DEFINITION 2.14. An operator $A$ possesses differential homogeneity of order $s \in \mathbb{R}$ and we write $A \in D H(s)$ if $t^{s} \sigma_{t}^{-1} A \sigma_{t} \subset A$ for all $t>0$.

For the sake of simplicity, we shall always assume that the operators $\Gamma$ and $\Gamma^{*}$ possess differential homogeneity of order 1 .

We now introduce the following purely technical definition. Unfortunately, the corresponding restrictions are sufficiently strong.

DEFINITION 2.15. For $p \in[1, \infty]$, we say that a subset $X \subset L_{l}$, is a CalderónZygmund subset for $L_{p}$, and we write $X \in C Z(p)$ if an arbitrary $f \in X$ admits a Calderón-Zygmund decomposition in terms of elements of $X$ at every level $\lambda>0$, that is, for some constants $C_{1}, C_{2}, C_{3}>0$ and every $f \in X$ and $\lambda>0$, there is a representation $f=f_{\infty}+\sum_{i \in \Im} f_{i}$ satisfying, for every $i \in \mathbb{N}$, supp $f_{i} \subset Q_{i}$ and $\left\|\left.f_{i}\left|L_{p}\left(Q_{i}\right) \| \leq \lambda C_{1}\right| Q_{i}\right|^{1 / p}\right.$, where $\left\{Q_{i}\right\}_{i \in \checkmark}$ is a system of cubes with the finite intersection property and $\sum_{i}\left|Q_{i}\right| \leq C_{2}\left\|f \mid L_{p}\right\|^{\prime \prime} / \lambda$, and $\left\|f_{\infty} \mid L_{\infty}\right\| \leq C_{3} \lambda$.

In addition to the classical example $L_{p} \in C Z(p)$, there are some other simple sufficient conditions.

REMARK 4. (a) If $X$ is a subspace of $L_{l}$, with inherited norm, and the projection $M_{\chi_{Q}}: f \mapsto \chi_{Q} f$ is bounded in $X$ for any cube $Q \subset \mathbb{R}^{n}$, then $X \in C Z(p)$ (see [2]).

(b) The assertion of (a) remains true if the pointwise multiplier $M_{\psi}$ is bounded in $X$ for any $\psi \in C_{0}^{\infty}$ instead of $\psi \in\left\{\chi_{Q}\right\}_{Q \subset R^{n}}$. It can be proved as in (a).

(c) If $X$ is complemented in $L_{p}$ under the conditions of (a), then the image $M_{\chi Q} X$ is complemented in $L_{p}$ uniformly by $Q \subset \mathbb{R}^{n}$. This observation provides an alternative proof of Lemma 4.8 with the aid of Lemma 4.9 and Remark 2 (1). 


\section{Main results}

Here we present the main results of the article in an abstract, but not the most general form. For the convenience of the reader a discussion of two further ways of generalizations is postponed until the beginning of Section 7 .

3.1. Functional calculus Here we state the results regarding the relationships between the existence of the functional calculus and the other basic properties defined in Section 2.

Some variations in part ( 3 ) of the conditions of the next two theorems are suggested by Corollary 4.4(a)-(b).

The following theorem is a generalization of Theorem 3.1, Chapter IV from [2], where the case of an injective $\Pi_{B}$ was considered.

THEOREM 3.1. For $0 \leq \omega<\nu<\mu<\pi / 2$ and $\emptyset \neq\left[q, q_{0}\right] \subset(1,2)$, assume that, along with $\mathbf{F}\left(\Pi_{B}, L_{2}, L_{2}, S_{\omega}^{d}\right), L_{2, \rho_{i, w^{\prime}}^{M}}=N_{2 . \rho_{l, w^{\prime}}^{M}}\left(\Pi_{B}\right) \oplus \overline{R_{2 . \rho_{i, w^{\prime}}^{M}}\left(\Pi_{B}\right)}$ holding uniformly for $t>0$ and $w \in \mathbb{R}^{n}, P_{0, q \mid N_{2, P_{1,1}^{M},}\left(\Pi_{B}\right)}=P_{0,2 \mid N_{4}\left(\Pi_{B}\right)}$,

$$
\left\{R\left(z e^{ \pm i \prime \prime} \cdot\right)\right\}_{z \in S_{0}^{d}} \subset \bar{D}\left(\Pi_{B}, S_{0}^{d}, 2,2, M, R_{2}\left(\Pi_{B}\right)\right) \quad \text { for any } M>0 \text {, and }
$$

$\overline{R_{r}\left(\Pi_{B}\right)} \in C Z(r)$ for some $r \in\left[q_{0}, 2\right)$, one of the following groups of conditions and the homogeneity property $\mathbf{H}[\mathbf{Z}]$ with respect to every condition $\mathbf{Z}$ in this group are satisfied:

(1) $L_{2}=N_{2}\left(\Pi_{B}\right) \oplus \overline{R_{2}\left(\Pi_{B}\right)}, L_{q_{v}}=N_{q_{0}}\left(\Pi_{B}\right) \oplus \overline{R_{q_{v}}\left(\Pi_{B}\right)}, \mathbf{S}\left(\Pi_{B}, q_{0}, 2, X\right)$, and $\Pi_{B}(X)$ is $L_{q_{0}}$-dense in $\overline{R_{40}\left(\Pi_{B}\right)}$;

(2) $\left(q_{s}, 2\right) \in \mathscr{S}_{1}, q_{0}=\max \left(q, q_{s}\right), \mathbf{G}(q), \mathbf{G}(2), \mathbf{C}, \mathbf{K}(q), \mathbf{P}(q), \mathbf{P}(2), \mathbf{W P}, \mathbf{D}(2)$, LC;

(3) $\left(q_{s}, 2\right) \in \mathscr{S}_{1}, q_{0}=\max \left(q, q_{s}\right), \mathbf{B C}(q), \mathbf{B C}(2), \mathbf{D}(q), \mathbf{D}(2), \mathbf{K}(q), \mathbf{C}, \mathbf{W P}, \mathbf{L C}$, $\overline{D_{q}\left(\Pi_{B}\right)}=L_{q}, \overline{D_{2}\left(\Pi_{B}\right)}=L_{2}$, and

$$
\begin{aligned}
\overline{R_{p^{\prime}}\left(\Gamma^{*}\right)} \cap N_{p^{\prime}}\left(\Gamma_{B^{*}}\right) & =N_{p^{\prime}}\left(\Gamma^{*}\right) \cap \overline{R_{p^{\prime}}\left(\Gamma_{B^{*}}\right)}=\overline{R_{2}\left(\Gamma^{*}\right)} \cap N_{2}\left(\Gamma_{B^{*}}\right) \\
& =N_{2}\left(\Gamma^{*}\right) \cap \overline{R_{2}\left(\Gamma_{B^{*}}\right)}=\{0\} .
\end{aligned}
$$

Then one has $\mathbf{F}\left(\Pi_{B}, L_{p}, L_{p}, S_{\mu}^{d}\right)$ for all $p \in\left(q_{0}, 2\right)$, that is

$$
\left\|f\left(\Pi_{B}\right) \phi\right\|_{p} \leq C\left\|f \mid \bar{H}_{\infty}\left(S_{\mu}^{d} \cup\{0\}\right)\right\| \cdot\|\phi\|_{p} \quad \text { for all } f \in \bar{H}_{\infty}\left(S_{\mu}^{d} \cup\{0\}\right), \phi \in L_{p},
$$

where $C$ depends on $p$.

PROOF. Let us begin with the observation that due to the construction of the functional calculus, it is sufficient to show its existence from $L_{p} \cap R_{p}\left(\Pi_{B}\right)$ into $L_{p}$ only, and 
that the main tool of the proof of Theorem 5.1, the Calderón-Zygmund decomposition is accessible due to the inclusion $\overline{R_{r}\left(\Pi_{B}\right)} \in C Z(r)$.

Then, it is enough to deal only with $f \in \Xi\left(S_{\mu}^{d}\right)$ thanks to the considerations from the classical density lemmas, namely, Lemma $D$ from [4] and Lemma 2 from [14]. The Cauchy representations used in the proofs of these density lemmas and in Theorems 4.6 and 4.14 (used in tandem as one rigorous assertion) can be deduced as in these sources because, as noticed above, the bounded $H_{\infty}\left(S_{\omega}^{d}\right)$-functional calculus implies the type $\omega$, and we can follow the reasoning from $[4,14]$ using the function $\psi(z)=z /\left(1+z^{2}\right)$ instead of the choice $\psi(z):=z /(1+z)^{2}$ made there. (The range of $R(\psi(A))=D(A)$ is still dense in $X$.)

The assertion of the theorem will follow from Theorem 5.1 if we check the validity of the conditions of that theorem for $T=f\left(\Pi_{B}\right), A_{t}=R\left(t z \Pi_{B}\right)$ for some $z \in \mathbb{C} \backslash S_{\mu}^{d}$ and sufficiently large $l$. The last condition is ensured by the assumption $\mathbf{F}(2)$. Then either Theorems 4.6, 4.14(a) and Lemma 4.7(a) in the case of the group (1), or the same results and Lemma 4.5(a) in the case of the second group, or Theorems 4.6 and 4.14(a), along with Lemmas 4.5(a), 4.7(a) and 4.3(a), and Corollary 4.4(a) in the case of the group (3) imply, thanks to Remark 2(1) that

$$
b^{k n} t^{\frac{n}{\rho}-\frac{n}{2}}\left\|f\left(\Pi_{B}\right) \Delta^{\prime} R\left(t z \Pi_{B}\right) \phi\left|L_{2}\left(Q_{b^{k+1} \delta}(w) \backslash Q_{b^{k} \delta \delta}(w)\right)\|\leq C\| f\right| H_{\infty}\right\| b^{k(n-N)}\|\phi\|_{p}
$$

for any $t>0, w \in \mathbb{R}^{n}$ and $\phi \in R_{p}\left(\Pi_{B}\right)$ with supp $\phi \subset Q_{t}(w)$, where one can choose $N>n$. Whence, we see that condition (1) of Theorem 5.1 is satisfied also. In the same manner, the validity of condition (2) is a consequence of the following estimates provided by either Theorem 4.14(a) and Lemma 4.7(a) in the case of the group (1), or the same results and Lemma 4.5(a) in the case of the second group, or Theorem 4.14(a), along with Lemmas 4.5(a), 4.7(a) and 4.3(a),(c), and Corollary 4.4(a) in the case of the group (3):

$$
\begin{aligned}
b^{k n} t^{n / p-n / 2}\left\|R\left(t z \Pi_{B}\right) \mid L_{2}\left(Q_{h^{k+1} t_{\delta}}(w) \backslash Q_{h^{k} \delta}(w)\right)\right\| & \leq C\left\|f \mid H_{\infty}\right\| b^{k\left(n-N_{1}\right)}\|\phi\|_{p} \\
t^{n / p-n / 2}\left\|R\left(t z \Pi_{B}\right) \mid L_{2}\left(Q_{t \delta}(w)\right)\right\| & \leq C\|\phi\|_{p}
\end{aligned}
$$

for some $N_{1}>n$ and any $t>0, w \in \mathbb{R}^{n}$ and $\phi \in R_{p}\left(\Pi_{B}\right)$ with supp $\phi \subset Q_{t}(w)$.

THEOREM 3.2. For $0 \leq \omega<v<\mu<\pi / 2$ and $\emptyset \neq\left[q_{0}, q\right] \subset(2, \infty)$, assume that, along with $\mathbf{F}\left(\Pi_{B}, L_{2}, L_{2}, S_{\omega}^{d}\right)$ and $\left\{R\left(z e^{ \pm i^{\prime}} \cdot\right)\right\}_{z \in S_{0}^{d}} \subset \bar{D}\left(\Pi_{B}, S_{0}^{d}, 2,2, M, R_{2}\left(\Pi_{B}\right)\right)$ for any $M>0$, one of the following groups of conditions and the homogeneity property $\mathbf{H}[\mathbf{Z}]$ with respect to every condition $\mathbf{Z}$ in this group are satisfied:

(1) $L_{q_{0}}=N_{q_{0}}\left(\Pi_{B}\right) \oplus \overline{R_{q_{0}}\left(\Pi_{B}\right)}, L_{2}=N_{2}\left(\Pi_{B}\right) \oplus \overline{R_{2}\left(\Pi_{B}\right)}, \mathbf{S}\left(\Pi_{B}, 2, q_{0}, X\right)$, and $\Pi_{B}(X)$ is $L_{2}$-dense in $\overline{R_{2}\left(\Pi_{B}\right)}$;

(2) $\left(2, q_{s}\right) \in \mathscr{S}_{1}, q_{0}=\min \left(q_{s}, q\right), \mathbf{G}^{*}(q), \mathbf{G}^{*}(2), \mathbf{D}(2), \mathbf{C}, \mathbf{K}^{*}(q), \mathbf{P}^{*}(q), \mathbf{P}^{*}(2)$, WP, LC; 
[13] Extrapolation of functional calculus, embedding theorems and Littlewood-Paley inequalities 309

(3) $\left(2, q_{s}\right) \in \mathscr{S}_{1}, q_{0}=\min \left(q_{s}, q\right), \mathbf{B C}^{*}(q), \mathbf{B C}^{*}(2), \mathbf{D}(q), \mathbf{D}(2), \mathbf{C}, \mathbf{K}^{*}(q), \mathbf{W P}$, LC, $\overline{D_{q^{\prime}}\left(\Pi_{B^{*}}^{*}\right)}=L_{q^{\prime}}, \overline{D_{2}\left(\Pi_{B^{*}}^{*}\right)}=L_{2}$, and

$\overline{R_{p}(\Gamma)} \cap N_{p}\left(\Gamma_{B}^{*}\right)=N_{p}(\Gamma) \cap \overline{R_{p}\left(\Gamma_{B}^{*}\right)}=\overline{R_{2}(\Gamma)} \cap N_{2}\left(\Gamma_{B}^{*}\right)=N_{2}(\Gamma) \cap \overline{R_{2}\left(\overline{\left.\Gamma_{B}^{*}\right)}\right.}=\{0\}$.

Then one has $\mathbf{F}\left(\Pi_{B}, L_{p}, L_{p}, S_{\mu}^{d}\right)$ for all $p \in\left(2, q_{0}\right)$, that is

$$
\left\|f\left(\Pi_{B}\right) \phi\right\|_{p} \leq C\left\|f \mid \bar{H}_{\infty}\left(S_{\mu}^{d} \cup\{0\}\right)\right\| \cdot\|\phi\|_{p}
$$

for all $f \in \bar{H}_{\infty}\left(S_{\mu}^{d} \cup\{0\}\right), \phi \in L_{p}$, where $C$ depends on $p$.

PROOF. Firstly let us repeat the observations made in the first two paragraphs of the proof of Theorem 3.1.

The assertion of the theorem follows now from Theorem 5.2 if we check the validity of the conditions (1), (2) and $T \in \mathscr{L}\left(L_{2}\right)$ of that theorem for $T=f\left(\Pi_{B}\right)$, $A_{t}=R\left(t z \Pi_{B}\right)$ for some $z \in \mathbb{C} \backslash S_{\mu}^{d}$ and sufficiently large $l$. The last condition is ensured by the assumption $\mathbf{F}(2)$. Then Theorem 4.6 implies, thanks to Remark 5 ,

$$
\begin{aligned}
t^{-n / 2}\left\|\Delta^{\prime} R\left(t z \Pi_{B}\right) f\left(\Pi_{B}\right) \phi \mid L_{2}\left(Q_{t}(w)\right)\right\| & \leq C\left\|f\left|H_{\infty}\|\cdot\| \phi\right| L_{2 . \rho_{i . .1}^{M} \|}\right\| \\
& \leq C\left\|f \mid H_{\infty}\right\| M_{2} \phi(w),
\end{aligned}
$$

for any $t>0, \phi \in R_{2}\left(\Pi_{B}\right)$ and a.e. $w \in \mathbb{R}^{n}$, where one can choose $M>n$. Having in mind that $L_{2}=N_{2}\left(\Pi_{B}\right) \oplus \overline{R_{2}\left(\Pi_{B}\right)}$ in the case of an arbitrary group of conditions (see Lemma 4.3(b),(d) and Corollary 4.4(b)), we can follow the definition of the functional calculus to show (3.1) for $\phi \in L_{2}$. Whence, we see that the condition (1) of Theorem 5.1 is satisfied too. In the same manner, the validity of the condition (2) of that theorem is a consequence of the following estimates provided by either Theorems 4.14 (b), Lemma 4.7 (b) in the case of the group (1), or the same results and Lemma 4.5(b) in the case of the second group, or Theorem 4.14(b), along with Lemmas 4.5(b), 4.7(b), 4.3(b) and 4.3(d) and Corollary 4.4(b) in the case of the group (3):

$$
t^{-n / p}\left\|R\left(t z \Pi_{B}\right) \phi\left|L_{2}\left(Q_{t}(z)\right)\|\leq C\| f\right| H_{\infty}\right\| \cdot\left\|\phi\left|L_{2, \rho_{1, k}^{M}}\|\leq C\| f\right| H_{\infty}\right\| M_{2} \phi(w)
$$

for some $M>n$ and any $t>0, \phi \in L_{2}$, where we can take $\phi \in L_{2}$ as it is done for the case of (1).

3.2. Generalized embedding theorems In this section, we study the boundedness properties of powers of the operator $\Pi_{B}$ from Besov, or Lizorkin-Triebel spaces into Lorentz, or Lebesgue spaces, correspondingly.

THEOREM 3.3. Under the conditions of either Theorem 3.1 or Theorem 3.2 for some $r \in(0, p], \theta \in(0, \infty), \beta \in(0, s]$ with $(s-\beta) / n=r^{-1}-p^{-1}$, assume 
$\Gamma, \Gamma^{*} \in D H(1)$ and either $s \in(0,1)$ and $Y_{r}^{s}=l_{r, \theta}^{s}$, or $s=1$ and $Y_{r}^{s}=w_{r}^{1}$. Then we have

$$
\Pi_{B}^{\beta} \in \mathscr{L}\left(\dot{Y}_{r}^{s}, L_{p}\right)
$$

Using real interpolation, we obtain the following.

COROLLARY 3.4. Under the conditions of Theorem 3.3, one also has

$$
\Pi_{B}^{\beta} \in \mathscr{L}\left(\dot{b}_{r, \theta}^{s}, L_{p, \theta}\right) .
$$

PROOF. Let us choose an entire function $\zeta \in \Xi\left(S_{\mu}^{\prime}\right)$ with $\xi(0)=0$. For $\Pi_{B}^{\beta}$, let us use the representation

$$
z^{\beta}=\int_{0}^{\infty} z^{\beta} \zeta(t z) \frac{d t}{t}
$$

Without loss of generality, we can assume also that

$$
\xi(z):=\int_{1}^{\infty}(t z)^{\beta} \zeta(t z) \frac{d t}{t} \in H^{\infty}\left(S_{\mu}\right)
$$

Then Theorem 3.1 shows that $\xi\left(\Pi_{B}\right) \in \mathscr{L}\left(L_{p}, L_{p}\right)$. Therefore, involving embedding properties of Sobolev and Lizorkin-Triebel spaces into Lebesgue spaces, we see also that $\xi\left(\Pi_{B}\right) \in \mathscr{L}\left(\dot{Y}_{r}^{s}, L_{p}\right)$. The identity

$$
\left(\xi\left(\Pi_{B_{\tau}}\right) f\right)(\cdot / \tau)=\xi\left(\tau \Pi_{B}\right) f(\cdot / \tau)
$$

and the homogeneity of $\dot{Y}_{r}^{s}$ show the uniform boundedness

$$
\left\|\tau^{-\beta} \xi\left(\tau \Pi_{B}\right)\left|\mathscr{L}\left(\dot{Y}_{r}^{s}, L_{p}\right)\|=\| \xi\left(\Pi_{B}\right)\right| \mathscr{L}\left(\dot{Y}_{r}^{s}, L_{p}\right)\right\| \quad \text { for every } \tau>0 .
$$

Thus, a choice of $\zeta$ well decaying at 0 finishes the proof with the aid of the identity

$$
\Pi_{B}^{\beta}=\int_{0}^{\infty} \Pi_{B}^{\beta} \zeta\left(t \Pi_{B}\right) \frac{d t}{t}=\lim _{\tau \rightarrow+0} \int_{\tau}^{\infty} \Pi_{B}^{\beta} \zeta\left(t \Pi_{B}\right) \frac{d t}{t}=\lim _{\tau \rightarrow+0} \tau^{-\beta} \xi\left(\tau \Pi_{B}\right) .
$$

3.3. Quadratic estimates and theorems of the Littlewood-Paley type. The next theorem is obtained in [14].

THEOREM 3.5. For $p \in(1, \infty)$, assume that $\mathbf{F}\left(A, p, S_{\mu}^{d}\right)$ holds. Then, for every $\phi \in Q\left(S_{\mu}^{d}\right)$, one has

(a) $\left\|\left(\int_{0}^{\infty}|\phi(t A) f|^{2} d t / t\right)^{1 / 2}\right\|_{p} \leq C_{f}(\phi)\|f\|_{p}$;

(b) $\left\|\int_{0}^{\infty} \phi\left(t A^{*}\right) f(t, \cdot) d t / t\right\|_{p^{\prime}} \leq C_{b}(\phi)\left\|\left(\int_{0}^{\infty}|f(t, \cdot)|^{2} d t / t\right)^{1 / 2}\right\|_{p^{\prime}}$,

if $A^{*}$ is the dual of $A$ in the pair $\left(L_{p^{\prime}}, L_{p}\right)$. 
[15] Extrapolation of functional calculus, embedding theorems and Littlewood-Paley inequalities 311

The next theorem follows from Theorems 3.1 and 3.5.

THEOREM 3.6. Assume the hypotheses of Theorem 3.1. Then, for every $\phi \in Q\left(S_{\mu}^{d}\right)$ and $p \in\left(q_{0}, 2\right)$, one has

(a) $\left\|\left(\int_{0}^{\infty}\left|\phi\left(t \Pi_{B}\right) f\right|^{2} d t / t\right)^{1 / 2}\right\|_{p} \leq C_{f}(\phi)\|f\|_{p}$;

(b) $\left\|\int_{0}^{\infty} \phi\left(t \Pi_{B^{*}}^{*}\right) f(t, \cdot) d t / t\right\|_{p^{\prime}} \leq C_{b}(\phi)\left\|\left(\int_{0}^{\infty}|f(t, \cdot)|^{2} d t / t\right)^{1 / 2}\right\|_{p^{\prime}}$,

if $\mathbf{D}(p)$ holds too.

The following theorem is a consequence of Theorems 3.2 and 3.5 .

THEOREM 3.7. Assume the hypotheses of Theorem 3.2. Then, for every $\phi \in Q\left(S_{\mu}^{d}\right)$ and $p \in\left(2, q_{0}\right)$, one has

(a) $\left\|\left(\int_{0}^{\infty}\left|\phi\left(t \Pi_{B}\right) f\right|^{2} d t / t\right)^{1 / 2}\right\|_{p} \leq C_{f}(\phi)\|f\|_{p}$;

(b) $\left\|\int_{0}^{\infty} \phi\left(t \Pi_{B^{*}}^{*}\right) f(t, \cdot) d t / t\right\|_{p^{\prime}} \leq C_{b}(\phi)\left\|\left(\int_{0}^{\infty}|f(t, \cdot)|^{2} d t / t\right)^{1 / 2}\right\|_{p^{\prime}}$. if $\mathbf{D}(p)$ holds too.

Now we are in a position to prove a theorem of the Littlewood-Paley type in terms of the functional calculus. The next theorem is the combination of Theorems 3.6 and 3.7 .

THEOREM 3.8. Assume the hypotheses of both Theorem 3.1 and Theorem 3.2. Then, for every $\phi \in Q\left(S_{\mu}^{d}\right)$ and $p \in\left(q_{0}, q_{0}^{\prime}\right)$, one has
(a) $\left\|\left(\int_{0}^{\infty}\left|\phi\left(t \Pi_{B}\right) f\right|^{2} d t / t\right)^{1 / 2}\right\|_{p} \asymp\|f\|_{p}$;
(b) $\left\|\left(\int_{0}^{\infty}\left|\phi\left(t \Pi_{B^{*}}^{*}\right) f\right|^{2} d t / t\right)^{1 / 2}\right\|_{p} \asymp\|f\|_{p^{*}}$

\section{Off-diagonal estimates}

We begin this section with a high-order counterpart of the Hilbert identity. Then we study the relations between Bounded Coercivity and the $L_{p}$-space structure of the model, and between the latter and Sobolev property. Eventually we derive the key results on off-diagonal estimates.

\subsection{Counterparts of the Hilbert identity}

\section{LEMMA 4.1.}

$$
\Delta^{k} R(z)=\frac{z^{k}}{\prod_{l=1}^{k}\left(l^{-1}-z\right)}, \quad k \in \mathbb{N}
$$


This simple identity suggests generalizations of the Hilbert identity for the differences of arbitrary high order in the form of Lemma 4.2.

Lemma 4.2. For $h, z_{0} \in \mathbb{C}, m \in \mathbb{N}$, let $X$ be a linear space, and $A: X \rightarrow X$ be $a$ linear operator with existing inverse operators $\left\{\left(A-\left(z_{0}+i h\right) I\right)^{-1}\right\}_{i=0}^{m}$, or $\left\{R\left(\left(z_{0}+i h\right) A\right)\right\}_{i=0}^{m}$. Then, for

$$
\Delta_{h}^{m} \psi\left(z_{0}\right):=\Delta_{h}^{1} \Delta_{h}^{m-1} \psi\left(z_{0}\right), \quad \Delta_{h}^{1} \psi\left(z_{0}\right):=\psi\left(z_{0}+h\right)-\psi\left(z_{0}\right),
$$

one has, correspondingly

(a) $\Delta_{h}^{m} \psi\left(z_{0}\right):=m ! h^{m} \prod_{i=0}^{m}\left(A-\left(z_{0}+i h\right) I\right)^{-1} \quad$ for $\psi(z):=(A-z I)^{-1}$, or

(b) $\Delta_{h}^{m} \psi\left(z_{0}\right):=m !(h A)^{m} \prod_{i=0}^{m}\left(I-\left(z_{0}+i h\right) A\right)^{-1}$ for $\psi(z):=R(z A)$.

PROOF. Let us prove part (a). The proof of (b) is essentially the same and, thus, is omitted. Using induction on $m$, we see that, for $m=1$, (a) is the Hilbert identity. Assuming the validity of (a) for $m=k-1$, one sees

$$
\begin{aligned}
\Delta_{h}^{k} \psi\left(z_{0}\right) & =(k-1) ! h^{k-1}\left(\prod_{i=1}^{k}\left(A-\left(z_{0}+i h\right) I\right)^{-1}-\prod_{i=0}^{k-1}\left(A-\left(z_{0}+i h\right) I\right)^{-1}\right) \\
& =(k-1) ! h^{k-1} \prod_{i=1}^{k}\left(A-\left(z_{0}+i h\right) I\right)^{-1} \Delta_{k h}^{1} \psi\left(z_{0}\right) \\
& =k ! h^{k} \prod_{i=0}^{k}\left(A-\left(z_{0}+i h\right) I\right)^{-1} .
\end{aligned}
$$

4.2. Perturbed projectors Recall that $\Gamma$ and $\Gamma_{B}^{*}$ are assumed to be densely defined in $L_{p}$, and $\mathrm{I}$ holds.

Lemma 4.3. For $p \in[1, \infty]$, one has:

(a) $\mathbf{B C}(p)$ implies restricted $\mathbf{G}(p)$;

(b) $\mathbf{B C}^{*}(p)$ implies restricted $\mathbf{G}^{*}(p)$;

(c) $\mathbf{B C}(p)$ and $\mathbf{D}(p)$ imply $N_{p}(\Gamma) \oplus \overline{R_{p}\left(\Gamma_{B}^{*}\right)}$ and $\overline{R_{p}(\Gamma)} \oplus N_{p}\left(\Gamma_{B}^{*}\right)$ are subspaces in $L_{p}$;

(d) $\mathbf{B C}^{*}(p)$ and $\mathbf{D}(p)$ imply $N_{p^{\prime}}\left(\Gamma^{*}\right) \oplus \overline{R_{p^{\prime}}\left(\Gamma_{B^{*}}\right)}$ and $\overline{R_{p^{\prime}}\left(\Gamma^{*}\right)} \oplus N_{p^{\prime}}\left(\Gamma_{B^{*}}\right)$ are subspaces in $L_{p^{\prime}}$;

(e) $\mathbf{B C}(p)$, I and $\mathbf{D}(p)$ imply $\overline{R_{p}\left(\Pi_{B}\right)}=\overline{R_{p}(\Gamma)} \oplus \overline{R_{p}\left(\Gamma_{B}^{*}\right)}, N_{p}\left(\Pi_{B}\right)=N_{p}(\Gamma) \cap$ $N_{p}\left(\Gamma_{B}^{*}\right)$ and $\Pi_{B} \in \mathscr{C}\left(L_{p}\right)$; 
[17] Extrapolation of functional calculus, embedding theorems and Littlewood-Paley inequalities 313

(f) $\mathbf{B C}^{*}(p)$, I and $\mathbf{D}(p)$ imply $\overline{R_{p^{\prime}}\left(\Pi_{B^{*}}^{*}\right)}=\overline{R_{p^{\prime}}\left(\Gamma^{*}\right)} \oplus \overline{R_{p^{\prime}}\left(\overline{\Gamma_{B^{*}}}\right)}, \quad N_{p}\left(\Pi_{B^{*}}^{*}\right)=$ $N_{p^{\prime}}\left(\Gamma^{*}\right) \cap N_{p^{\prime}}\left(\Gamma_{B^{*}}\right)$ and $\Pi_{B^{*}}^{*} \in \mathscr{C}\left(L_{p^{\prime}}\right)$.

(g) For a Banach space $B$ and its subspaces $M, M_{0}, M_{1}, N, N_{0}, N_{1}$ satisfying $M=M_{0} \oplus M_{1}, N=N_{0} \oplus N_{1}$ and $M_{0} \supset N_{1}, N_{0} \supset M_{1}$, one has $M \cap N=$ $\left(M_{0} \cap N_{0}\right) \oplus M_{1} \oplus N_{1}$.

ProOF. Thanks to $\mathbf{B C}(p)$ and the Hölder inequality, for any $i$ and $f \in R_{p}\left(A_{i}\right)$, there is $g \in R_{p^{\prime}}\left(A_{i}\right)$, such that

$$
C\|f\|_{p}^{2} \leq C\left|b_{i}(g, f)\right| \leq\|g\|_{p^{\prime}} \cdot\left\|B_{i} f\right\|_{p} \leq\|f\|_{p} \cdot\left\|B_{i} f\right\|_{p} .
$$

Thus, $\left\{B_{i}\right\}_{i=1}^{2}$ are invertible on $\left\{\overline{R_{p}\left(A_{i}\right)}\right\}_{i=1}^{2}$ respectively. This proves (a). Similarly one obtains (b).

In the case of (c), thanks to $\mathbf{B C}(p)$, for any $f \in D_{p}\left(\Gamma_{B}^{*}\right)$, there is $g \in R_{p^{\prime}}\left(\Gamma^{*}\right)$ satisfying

$$
\left\|\Gamma^{*} g\right\|_{p^{\prime}} \leq C\left\|\Gamma^{*} B_{2} f\right\|_{p} \quad \text { and } \quad\left\|\Gamma^{*} B_{2} f\right\|_{p} \leq C\left|b_{1}\left(\Gamma^{*} g, \Gamma^{*} B_{2} f\right)\right| .
$$

Thus, with the aid of (4.2), $\mathbf{D}(p)$ and the Hölder inequality, one has, for any $h \in N_{p}(\Gamma)$,

$$
\left\|\Gamma^{*} B_{2} f\right\|_{p}^{2} \leq C\left|\int \overline{\Gamma^{*} g}\left(\Gamma_{B}^{*} f+h\right)\right| \leq C\left\|\Gamma^{*} g\right\|_{p^{\prime}} \cdot\left\|\Gamma_{B}^{*} f+h\right\|_{p} .
$$

Due to (a), it follows that

$$
\left\|\Gamma_{B}^{*} f\right\|_{p} \leq\left\|B_{1 \mid \overline{R_{p}\left(\Gamma_{B}^{*}\right)}}^{-1} \mathscr{L}\left(\overline{R_{p}\left(\Gamma_{B}^{*}\right)}, L_{p}\right)\right\| \cdot\left\|\Gamma^{*} B_{2} f\right\|_{p} \leq C\left\|\Gamma_{B}^{*} f+h\right\|_{p},
$$

showing that $N_{p}(\Gamma) \oplus \overline{R_{p}\left(\Gamma_{B}^{*}\right)}$ is a subspace in $L_{p}$. In the case of the second assertion of (c), we do not use (a).

$$
\begin{aligned}
C\|\Gamma f\|_{p}^{2} \leq\left|b_{2}(\Gamma g . \Gamma f)\right| & =\left|b_{2}(\Gamma g, \Gamma f+h)\right| \\
& \leq\left\|B_{2} \mid \mathscr{L}\left(L_{p}\right)\right\| \cdot\|\Gamma g\|_{p^{\prime}} \cdot\|\Gamma f+h\|_{p},
\end{aligned}
$$

where $h \in N_{p}\left(\Gamma_{B}^{*}\right)$ and $\|\Gamma g\|_{p^{\prime}} \leq C\|\Gamma f\|_{p}$.

In the case of $(\mathrm{d})$, we repeat the proof of the previous part with $\Gamma^{*}, \Gamma, B_{2}^{*}, B_{1}^{*}, b_{1}^{*}, b_{2}^{*}$ and $p^{\prime}$ in place of $\Gamma, \Gamma^{*}, B_{1}, B_{2}, b_{1}, b_{2}$ and $p$, respectively.

Thanks to I and Remark 2, we have $\overline{R_{p}(A)} \subset N_{p}(A)$ for any $A \in\left\{\Gamma, \Gamma^{*}, \Gamma_{B}^{*}, \Gamma_{B^{*}}\right\}$. This means that (e) and (f) follow from (c) and (d) correspondingly.

Under the hypothesis of $(\mathrm{g})$, we have $M_{1} \subset N$, hence, $M \cap N=\left(M_{0} \cap N\right) \oplus M_{1}$. In the same way, the inclusion $N_{1} \subset M_{0}$ is followed by $M_{0} \cap N=\left(M_{0} \cap N_{0}\right) \oplus N_{1}$ implying the assertion of $(\mathrm{g})$.

COROLlaRY 4.4. For $p \in(1, \infty)$, assume that $\mathbf{D}(p)$, I and one of the following groups of conditions hold: 
(a) $\mathbf{B C}(p)$ and either $\overline{R_{p^{\prime}}\left(\Gamma^{*}\right)} \cap N_{p^{\prime}}\left(\Gamma_{B^{*}}\right)=N_{p^{\prime}}\left(\Gamma^{*}\right) \cap \overline{R_{p^{\prime}}\left(\Gamma_{B^{*}}\right)}=\{0\}$, or $\overline{D_{p}\left(\Pi_{B}\right)}=L_{p}, N_{p^{\prime}}\left(\Pi_{B^{*}}^{*}\right) \cap \overline{R_{p^{\prime}}\left(\Pi_{B}^{*}\right)}=\{0\}$;

(b) $\mathbf{B C}^{*}(p)$ and either $\overline{R_{p}(\Gamma)} \cap N_{p}\left(\Gamma_{B}^{*}\right)=N_{p}(\Gamma) \cap \overline{R_{p}\left(\Gamma_{B}^{*}\right)}=\{0\}$, or $\overline{D_{p}\left(\Pi_{B}\right)}=L_{p^{\prime}}, N_{p^{\prime}}\left(\Pi_{B^{*}}^{*}\right) \cap \overline{R_{p^{\prime}}\left(\Pi_{B^{*}}^{*}\right)}=\{0\}$;

(c) $\mathbf{B C}(p)$ and $\mathbf{B C}^{*}(p)$.

Then $\mathbf{P}(p)$ and $\mathbf{P}^{*}(p)$ hold.

Proof. To prove (a), observe that $\mathbf{D}(p)$, the closedness of $\Gamma$ and $\Gamma_{B}^{*}$ and the reflexivity of $L_{p}$ imply

$$
\begin{gathered}
N_{p}(\Gamma)^{\perp}=\overline{R_{p^{\prime}}\left(\Gamma^{*}\right)}, \quad N_{p}\left(\Gamma_{B}^{*}\right)^{\perp}=\overline{R_{p^{\prime}}\left(\Gamma_{B^{*}}\right)}, \quad \overline{R_{p}(\Gamma)}{ }^{\perp}=N_{p^{\prime}}\left(\Gamma^{*}\right) \\
\text { and } \overline{R_{p}\left(\Gamma_{B}^{*}\right)}{ }^{\perp}=N_{p^{\prime}}\left(\Gamma_{B^{*}}\right)
\end{gathered}
$$

followed by the relations

$$
\begin{gathered}
\left(N_{p}(\Gamma)+\overline{R_{p}\left(\Gamma_{B}^{*}\right)}\right)^{\perp}=N_{p^{\prime}}\left(\Gamma_{B^{*}}\right) \cap \overline{R_{p^{\prime}}\left(\Gamma^{*}\right)}, \\
\left(N_{p}\left(\Gamma_{B}^{*}\right)+\overline{R_{p}(\Gamma)}\right)^{\perp}=N_{p^{\prime}}\left(\Gamma^{*}\right) \cap \overline{R_{p^{\prime}}\left(\Gamma_{B^{*}}\right)},
\end{gathered}
$$

where + means the algebraic sum. Thus. concerning the case

$$
\overline{R_{p^{\prime}}\left(\Gamma^{*}\right)} \cap N_{p^{\prime}}\left(\Gamma_{B^{*}}\right)=N_{p^{\prime}}\left(\Gamma^{*}\right) \cap \overline{R_{p^{\prime}}\left(\Gamma_{B^{*}}\right)}=\{0\}
$$

we have

$$
\begin{gathered}
L_{p}=N_{p}(\Gamma) \oplus \overline{R_{p}\left(\Gamma_{B}^{*}\right)}=N_{p}\left(\Gamma_{B}^{*}\right) \oplus \overline{R_{p}(\Gamma)}, \\
\overline{R_{p}(\Gamma)} \subset N_{p}(\Gamma) . \quad \overline{R_{p}\left(\Gamma_{B}^{*}\right)} \subset N_{p}\left(\Gamma_{B}^{*}\right),
\end{gathered}
$$

and, therefore, thanks to Lemma $4.3(\mathrm{~g})$,

$$
\begin{aligned}
N_{p}(\Gamma) & =\left(N_{p}(\Gamma) \cap N_{p}\left(\Gamma_{B}^{*}\right)\right) \oplus \overline{R_{p}(\Gamma)}, \\
N_{p}\left(\Gamma_{B}^{*}\right) & =\left(N_{p}\left(\Gamma_{B}^{*}\right) \cap N_{p}(\Gamma)\right) \oplus \overline{R_{p}\left(\Gamma_{B}^{*}\right),}
\end{aligned}
$$

because $N_{p}\left(\Pi_{B}\right)=N_{p}(\Gamma) \cap N_{p}\left(\Gamma_{B}^{*}\right)$ due to Lemma 4.3(e).

In the case $\overline{D_{p}\left(\Pi_{B}\right)}=L_{p}, N_{p^{\prime}}\left(\Pi_{B^{*}}^{*}\right) \cap \overline{R_{p^{\prime}}\left(\Pi_{B^{*}}^{*}\right)}=\{0\}$, we use Lemma 4.3(c)-(g) to observe

$$
\begin{aligned}
& \left(N_{p}(\Gamma) \oplus \overline{R_{p}\left(\Gamma_{B}^{*}\right)}\right) \cap\left(\overline{R_{p}(\Gamma)} \oplus N_{p}\left(\Gamma_{B}^{*}\right)\right) \\
& \quad=\left(N_{p}(\Gamma) \cap N_{p}\left(\Gamma_{B}^{*}\right)\right) \oplus\left(\overline{R_{p}\left(\Gamma_{B}^{*}\right)} \oplus \overline{R_{p}(\Gamma)}\right)=N_{p}\left(\Pi_{B}\right) \oplus \overline{R_{p}\left(\Pi_{B}\right)}
\end{aligned}
$$

Now, as in (4.6) and (4.7), the closedness of $\Pi_{B}$ following from Lemma 4.3(e) and the reflexivity of $L_{p}$ imply

$$
\left(N_{p}\left(\Pi_{B}\right) \oplus \overline{R_{p}\left(\Pi_{B}\right)}\right)^{\perp}=N_{p^{\prime}}\left(\Pi_{B^{*}}^{*}\right) \cap \overline{R_{p^{\prime}}\left(\Pi_{B^{*}}^{*}\right)}=\{0\},
$$


that is, $\mathbf{P}(p)$. Duality considerations and (4.6) provide $\mathbf{P}^{*}(p)$, if we follow the same procedure as before involving Lemma $4.3(\mathrm{~g})$.

The proof of (b) is conducted in the same way. Part (c) follows from (a) and (b) with the aid of Lemma 4.3(c),(d).

4.3. Sobolev properties For the sake of simplicity, we shall sometimes use symbols $\Pi_{B}^{-1}$ and $\Pi_{B^{*}}^{*-1}$ instead of $\left(\Pi_{B \mid \widehat{R_{p}\left(\Pi_{B}\right)}}\right)^{-1}$ and $\left(\Pi_{B^{*} \mid \overline{R_{p^{\prime}}}\left(\Pi_{B^{*}}^{*}\right)}\right)^{-1}$, respectively.

LEMMA 4.5. (a) For $q \in(1,2)$, assume that the conditions $\left(q_{s}, 2\right) \in \mathscr{S}_{1}, \mathbf{G}(q)$, $\mathbf{G}(2), \mathbf{C}, \mathbf{P}(q), \mathbf{P}(2), \mathbf{W P}, \mathbf{D}(2), \mathbf{L C}$ and $\mathbf{K}(q)$ hold. Then $\Pi_{B}^{-1}$ exists and extends to a bounded operator from $\overline{R_{p}\left(\Pi_{B}\right)}$ with $L_{p}$-metric into $L_{2}$ for all $p \in\left[\max \left(q_{s}, q\right), 2\right)$.

(b) For $q \in(2, \infty)$, assume conditions $\left(2, q_{s}\right) \in \mathscr{S}_{1}, \mathbf{G}^{*}(q), \mathbf{G}^{*}(2), \mathbf{D}(2), \mathbf{C}, \mathbf{P}^{*}(q)$, $\mathbf{P}^{*}(2), \mathbf{W P}, \mathbf{L C}$ and $\mathbf{K}^{*}(q)$. Then $\Pi_{B}^{-1}$ exists and extends to a bounded operator from $\overline{R_{2}\left(\Pi_{B}\right)}$ with $L_{2}$-metric into $L_{p}$ for all $p \in\left(2, \min \left(q_{s}, q\right)\right]$.

PROOF. In the case of part (a), let us designate $q_{0}=\max \left(q_{s}, q\right)$. Thanks to Corollaries 4.11(a) and 4.10, it is sufficient to consider the case $p=q_{0}$ only. If $q_{s}>q$, then we use Corollary 4.11 (a) to obtain $\mathbf{P}\left(q_{s}\right)$. Otherwise, one has $(q, 2) \in \mathscr{S}_{1}$. In both cases, $\mathbf{P}\left(q_{0}\right)$ is followed by $\overline{R_{q_{0}}\left(\Pi_{B}\right)}=\overline{R_{q_{0}}(\Gamma)} \oplus \overline{R_{q_{0}}\left(\Gamma_{B}^{*}\right)}$ in algebraic and topological senses. While $\mathbf{G}(q), \mathbf{G}(2)$ and Corollary $4.11(\mathrm{~b})$ imply $\overline{R_{q 0}\left(\Gamma_{B}^{*}\right)} \subset B_{1} \overline{R_{q_{0}}\left(\Gamma^{*}\right)}$.

Therefore, we can prove the assertion of the part (a) of the lemma on each of the subspaces $\overline{R_{q_{0}}(\bar{\Gamma})}$ and $\overline{R_{q_{0}}\left(\Gamma_{B}^{*}\right)}$ separately. These considerations and the idempotence condition (I) imply

$$
\begin{gathered}
\Pi_{B}^{-1} g=\left(\Pi_{B}^{-1} \Gamma\right) \Pi^{-1} g \quad \text { for all } g \in R_{q_{0}}(\Gamma), \text { and, for all } g \in R_{q_{0}}\left(\Gamma_{B}^{*}\right) \\
h=\Pi_{B}^{-1} B_{1} \Gamma^{*} \Pi^{-1} B_{1}^{-1} h=\left(\Pi_{B}^{-1} \Gamma_{B}^{*}\right)\left(B_{2}^{-1} \Pi^{-1} B_{1}^{-1}\right) h
\end{gathered}
$$

because of the identities $\Gamma \Pi^{-1} g=g$ for all $g \in R_{q_{0}}(\Gamma)$ and $B_{1} \Gamma \Pi^{-1} B_{1}^{-1} g=g$ for all $g \in B_{1} R_{q_{0}}\left(\Gamma^{*}\right)$ and the injectivity of $\Pi_{B}$ on $\overline{R_{q_{0}}\left(\Pi_{B}\right)}$ following from $\mathbf{P}\left(q_{0}\right)$. The duality condition $\mathbf{D}(2)$ implies that $\Pi=\Pi^{*}$ is self-adjoint in $L_{2}$ and, thus, on $D_{2}(\Pi) \cap \overline{R_{2}(\Pi)}$, which is $L_{2}$-dense in $\overline{R_{2}(\Pi)}$. Hence, $\Pi^{-1}$ exists on $R_{2}(\Pi)$ due to the weak projectivity condition (WP) and is self-adjoint too. The inclusion of $\left(q_{0}, 2\right)$ into the Sobolev relation $\mathscr{S}_{1}$, the coercivity property $(\mathbf{C})$ and continuity considerations show that $\left(\Pi^{-1}\right)^{*}=\Pi^{-1}$ extends to a bounded operator from $\overline{R_{2}(\Pi)}$ with $L_{2}$-metric into $L_{q_{0}^{\prime}}$ and into $L_{2}$. Therefore, for $f \in \overline{R_{2}(\Pi)} \cap L_{q_{0}}$ and $g \in \overline{R_{2}(\Pi)}$, with the aid of the Hölder inequality, one has

$$
\begin{aligned}
\left|\int \bar{g} \Pi^{-1} f\right| & =\left|\int \overline{\Pi^{-1} g} f\right| \leq C\left\|\Pi^{-1} g\left|L_{q_{0}^{\prime}}\|\| f\right| L_{q_{0}}\right\| \\
& \leq C\left\|g\left|L_{2}\|\| f\right| L_{q_{0}}\right\| .
\end{aligned}
$$


Thus, having in mind that $R_{2}(\Pi)$ is a norming set for $\overline{R_{2}(\Pi)}$, and continuity considerations, we see with the aid of the local closure condition (LC) that $\Pi^{-1}$ extends to a bounded operator from $\overline{R_{q_{0}}(\Pi)}$ with $L_{q_{0}}$-metric into $L_{2}$. Now Corollary 2.8 , along with $\mathbf{P}(2)$, implies $\Pi_{B}^{-1} \Gamma \subset P_{2} \in \mathscr{L}\left(L_{2}\right)$ and $\Pi_{B}^{-1} \Gamma_{B}^{*} \subset P_{1} \in \mathscr{L}\left(L_{2}\right)$. The continuity considerations finish the proof of (a).

In the case of the part (b), let us observe that $\Pi_{B}$ is injective on $\overline{R_{2}\left(\Pi_{B}\right)}$ due to $\mathbf{P}(2)$ and $\mathbf{G}(2)$. Designating $q_{0}:=\min \left(q_{s}, q\right)$, we see that the boundedness of $\Pi_{B}^{-1}$ from $R_{2}\left(\Pi_{B}\right)$ with $L_{2}$-metric into $L_{q 0}$ would imply (b). Thanks to Corollaries 4.11(a) and 4.10 , it is sufficient to consider the case $p=q_{0}$ only. Let us also observe that $\mathbf{P}^{*}(2), \mathbf{P}^{*}(q)$ and $\mathbf{K}^{*}(q)$ imply $\mathbf{P}^{*}\left(q_{0}\right)$ thanks to Corollary $4.11(\mathrm{a})$.

We start with the investigation of the boundedness properties of $\Pi_{B^{*}}^{*-1}$. To accomplish this task, we utilize the considerations of the approach used in part (a), including the boundedness of $\Pi^{-1}$ from $\overline{R_{q_{0}^{\prime}}(\Pi)}$ with $L_{q_{0}^{\prime}}$-metric into $L_{2}$ following from $\mathbf{D}(2)$, WP, $\mathbf{L C}$ and $\left(2, q_{0}\right) \in \mathscr{S}_{1}$ : for all $g \in \Pi_{B^{*}}^{*}(D)$, one has

$$
\begin{aligned}
\Pi_{B^{*}}^{*-1} g & =\Pi_{B^{*}}^{*-1}\left(\Gamma^{*} \Pi^{-1}\right) P_{1.4_{0}^{\prime}}^{*} g+\Pi_{B^{*}}^{*-1}\left(B_{2}^{*} \Gamma \Pi^{-1} B_{2}^{*-1}\right) P_{2 . q_{0}^{\prime}}^{*} g \\
& =\left(\Pi_{B^{*}}^{*-1} \Gamma^{*}\right) \Pi^{-1} P_{1 . q_{0}}^{*} g+\left(\Pi_{B^{*}}^{*-1} \Gamma_{B^{*}}\right)\left(B_{1}^{*-1} \Pi^{-1} B_{2}^{*-1}\right) P_{2 . q_{0}^{\prime}}^{*} g,
\end{aligned}
$$

where the last identity is due to Corollary $4.11(\mathrm{c})$. Since we know from the proof of (a) that $\Pi^{-1}$ is bounded from $\overline{R_{q_{0}^{\prime}}(\Pi)}$ with $L_{q_{0}^{\prime}}$-metric into $L_{2}$, as it was done in (a), Corollary 2.8(b) provides the boundedness of $\Pi_{B^{*}}^{*-1}$ from $R_{q_{0}^{\prime}}\left(\Pi_{B^{*}}^{*}\right)$ with $L_{q_{0}^{\prime}}$-metric into $L_{2}$ thanks to $\mathbf{P}^{*}(2), \mathbf{P}^{*}\left(q_{0}\right)$. Similarly we see also the boundedness of $\Pi_{B^{*}}^{*-1}$ from $R_{2}\left(\Pi_{B^{*}}^{*}\right)$ with $L_{2}$-metric into $L_{2}$. In both cases, $\Pi_{B^{*}}^{*-1}$ admits unique extension by continuity. In addition, $\mathbf{D}(2)$ shows that $\Pi_{B}^{*-1}=\Pi_{B}^{-1 *}$. With the aid of the real interpolation, we observe that, due to Corollary $4.11(\mathrm{a}), \mathbf{K}^{*}(q), \mathbf{P}^{*}(2)$ and $\mathbf{K}^{*}(q)$

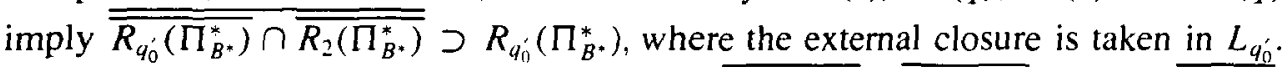

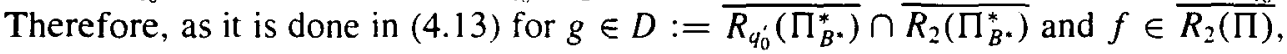
with the aid of the Hölder inequality. one has

$$
\left|\int \bar{g} \Pi_{B}^{-1} f\right|=\left|\int \overline{\Pi_{B^{*}}^{*-1} g} f\right| \leq C\left\|\Pi_{B^{*}}^{*-1} g\left|L_{2}\|\cdot\| f\right| L_{2}\right\| \leq C\left\|g\left|L_{q_{0}^{\prime}}\|\cdot\| f\right| L_{q_{0}}\right\| \text {. }
$$

4.4. Off-diagonal estimates The next theorem is a generalization of Theorem 4.2 from Chapter IV of [2].

THEOREM 4.6. Assume $\mu \in(0, \pi / 2), \nu \in(0, \mu), \Omega_{\mu}=\mathbb{C} \backslash S_{\mu}^{d}, k \in \mathbb{N}, p, q \in$ $[1, \infty], 0 \leq M<N$ and that $X \subset L_{p}$ is a linear subspace and $X_{1} \subset X \subset \bar{X}_{1}$. In addition, let $f \in \Xi\left(S_{\mu}^{d}\right),\left\|f \mid H_{\infty}\left(S_{\mu}\right)\right\| \leq 1$, and, for all $x \in X_{1}$, the Cauchy representation hold in $L_{q}$ :

$$
f(A) \Delta^{k} R(z A) x=\oint_{\gamma_{r}^{d}} f(\zeta) \Delta^{k} R(z \zeta) R\left(\zeta^{-1} A\right) x \frac{d \zeta}{\zeta} .
$$


[21] Extrapolation of functional calculus, embedding theorems and Littlewood-Paley inequalities 317

Then one has:

(a) $\left\{f(\cdot) \Delta^{k} R(z \cdot)\right\}_{z \in \Omega_{\mu}} \in \bar{D}\left(A, \Omega_{\mu}, p, q, X\right)$ for all $k>n / q-n / p>0$, if $\left\{R\left(z e^{ \pm i v} \cdot\right)\right\}_{z \in \bar{S}_{0}} \in \tilde{D}\left(A, S_{0}, p, q, X\right)$;

(b) $\left\{f(\cdot) \Delta^{k} R(z \cdot)\right\}_{z \in \Omega_{\mu}} \in \bar{D}\left(A, S_{v_{1}}, p, q, M, X\right)$

for $M / N<(k+n / p-n / q) /(k+N)$, if $\left\{R\left(z e^{ \pm i i^{\prime} \cdot}\right)\right\}_{z \in \bar{S}_{0}} \in \bar{D}\left(A, S_{0}, p, q, N, X\right)$ and $n / p-n / q \in(-k, N)$.

REMARK 5. (a) Theorem 4.6 is also true with $\Delta^{k} R(z \cdot) f(\cdot)$ taken instead of $f(\cdot) \Delta^{k} R(z \cdot)$.

(b) While the rigorous proof of the main theorems uses only the considerations from its proof combined with the proof of Theorem 4.14 in one assertion, we believe that Theorem 4.6 is of independent interest.

Proof. Thanks to the Minkowski inequality, uniformly by $z$ and $w \in \mathbb{R}^{n}$, we have, for any $\phi \in X_{1}$,

$$
\begin{aligned}
& \left\|f(A) \Delta^{k} R(z A) \phi\right\|_{q} \\
& \leq \int_{\gamma_{1}^{\prime},|z \zeta| \leq 1}\left|\Delta^{k} R(z \zeta)\right| \cdot\left\|R\left(\zeta^{-1} A\right) \phi\right\|_{q} \frac{|d \zeta|}{|\zeta|} \\
& +\int_{\gamma, \mid z \zeta>>1}\left|\Delta^{k} R(z \zeta)\right| \cdot\left\|R\left(\zeta^{-1} A\right) \phi\right\|_{q} \frac{|d \zeta|}{|\zeta|}=: I_{1}^{a}+I_{2}^{a} ; \\
& \left\|f(A) \Delta^{k} R(z A) \phi \mid L_{q}\left(Q_{|=|}(w)\right)\right\| \\
& \leq \int_{\gamma_{t}^{k}}\left|\Delta^{k} R(z \zeta)\right| \cdot\left\|R\left(\zeta^{-1} A\right) \phi \mid L_{\varphi}\left(Q_{|\equiv|}(w)\right)\right\| \frac{|d \zeta|}{|\zeta|}=I(\phi) .
\end{aligned}
$$

Now Lemma 4.1 and definitions permit us to finish the proof of part (a):

$$
\begin{aligned}
& \frac{I_{1}^{a}}{\|\phi\|_{p}} \leq C(A, k) k !(\sin |\mu-v|)^{-k}|z|^{k} \int_{0}^{k||^{-1}} r^{k+n / p-n / q-1} d r=C|z|^{n / q-n / p}, \\
& \frac{I_{2}^{a}}{\|\phi\|_{p}} \leq C(A, k) \int_{|z|^{-1}}^{\infty} r^{n / p-n / q-1} d r=C|z|^{n / q-n / p} .
\end{aligned}
$$

To proceed with (b), let us take some $b>0$ and denote $\Omega_{0 . t}(w):=Q_{t}(w)$ and $\Omega_{i, t}(w):=Q_{b^{i} t}(w) \backslash Q_{b^{i-1} t}(w)$ for $i \in \mathbb{N}, t>0$ and $w \in \mathbb{R}^{n}$. Assume also that $\tau=|z \zeta|^{-1}, \alpha:=n / p-n / q$ and $\varepsilon=N(k+\alpha) /(k+N)-M>0$. As above, we use the consequence of Lemma 4.1

$$
\left|\Delta^{k} R(z \zeta)\right| \leq C(A, k) \min \left(1, \tau^{-k}\right)
$$


the embedding $l_{1} \subset l_{p}$ and a Hölder inequality to derive the key relations

$$
\begin{aligned}
& |z|^{n / p-n / q} I(\phi) \\
& \leq C \int_{0}^{\infty} \min \left(1, \tau^{-k}\right)\left\|\phi \mid L_{p . \rho_{i=1 . u,}^{N}}\left(\mathbb{R}^{n}\right)\right\| \\
& \leq C \int_{0}^{\infty} \min \left(1, \tau^{-k}\right)\left(\sum_{i=0}^{\infty} \min \left(1,\left(\tau b^{-i}\right)^{p N}\right)\left\|\phi \mid L_{p}\left(\Omega_{i,|z|}(w)\right)\right\|^{p}\right)^{1 / p} \tau^{-\alpha-1} d \tau \\
& \leq C \sum_{i=0}^{\infty}\left\|\phi \mid L_{p}\left(\Omega_{i|| \xi \mid}(w)\right)\right\| \int_{0}^{\infty} \min \left(1, \tau^{-k}\right) \min \left(1,\left(\tau b^{-i}\right)^{N}\right) \tau^{-\alpha-1} d \tau \\
& =C \sum_{i=0}^{\infty} b^{-i(M+\varepsilon)}\left\|\phi\left|L_{p}\left(\Omega_{i,|\xi|}(w)\right)\left\|\leq C\left(\sum_{i=0}^{\infty} b^{-i p^{\prime} \varepsilon}\right)^{1 / p^{\prime}}\right\| \phi\right| L_{p, \rho_{i \mid 1,1}^{M}}\left(\mathbb{R}^{n}\right)\right\| .
\end{aligned}
$$

Combining the formulae (4.16) and (4.18), we finish the proof of the theorem.

LEMMA 4.7. (a) For $0 \leq \omega<\nu<\pi / 2$ and $q \in(1,2)$, assume the conditions $(q, 2) \in \mathscr{S}_{1}, \mathbf{S}\left(\Pi_{B}, q, 2, X\right)$, and that $\Pi_{B}(X)$ is $L_{q}$-dense in $\overline{R_{q}\left(\Pi_{B}\right)}$. Let, in addition, the set $\left\{R\left( \pm e^{ \pm i} \Pi_{B}\right)\right\}$ be in $\mathscr{L}\left(L_{2}\right)$. Assume also homogeneity property $\mathbf{H}$ with respect to all these conditions. Then we have $\left\{R\left(z e^{ \pm i "} \cdot\right)\right\}_{z \in S_{0}} \in \bar{D}\left(\Pi_{B}, S_{0}, q, 2, R_{q}\left(\Pi_{B}\right)\right)$.

(b) For $0 \leq \omega<\nu<\pi / 2$ and $q \in(2, \infty)$, assume the conditions $(2, q) \in \mathscr{S}_{1}$, $\mathbf{S}\left(\Pi_{B}, 2, q, X\right)$, and that $\Pi_{B}(X)$ is $L_{2}$-dense in $\overline{R_{2}\left(\Pi_{B}\right)}$. Let also the set $\left\{R\left( \pm e^{ \pm i \prime} \Pi_{B}\right)\right\}$ be in $\mathscr{L}\left(L_{2}\right)$. Assume, in addition, the homogeneity property $\mathbf{H}$ with respect to all these conditions. Then we have $\left\{R\left(z e^{ \pm i n} \cdot\right)\right\}_{z \in S_{0}} \in \bar{D}\left(\Pi_{B}, S_{0}, 2, q, R_{2}\left(\Pi_{B}\right)\right)$.

ProOF. Thanks to the homogeneity condition $\mathbf{H}$, it is sufficient to show the boundedness of operators $R(z A)$ for $|z|=1$ only. Due to the Sobolev conditions, $\Pi_{B}$ is injective on $X$ in both (a) and (b).

In the case of the part (a), let us utilise the identity

$$
\left(I-z \Pi_{B}\right)^{-1} g=\left(I-z \Pi_{B}\right)^{-1} \Pi_{B}\left(\Pi_{B}^{-1} g\right)=z^{-1}\left(R\left(z \Pi_{B}\right)-I\right)\left(\Pi_{B}^{-1} g\right)
$$

for all $g \in \Pi_{B}(X)$, the Sobolev condition and the inclusions $R\left( \pm e^{ \pm i v} \Pi_{B}\right) \in \mathscr{L}\left(L_{2}\right)$ to finish the proof of (a) in the view of the continuity considerations

$$
\overline{R_{q}\left(\Pi_{B}\right)} \supset \Pi_{B}(X) \stackrel{\Pi_{B}^{-1}}{\longrightarrow} L_{2} \stackrel{--1}{{ }^{-1}\left(I-R\left(Z \Pi_{B}\right)\right)} L_{2} .
$$

In the case of part (b), we start with the observation that, thanks to continuity considerations, $\mathbf{S}\left(\Pi_{B}, 2, q, R_{2}\left(\Pi_{B}\right)\right)$ holds. The identity

$$
\begin{aligned}
\left(I-z \Pi_{B}\right)^{-1} g & =\left(\Pi_{B \mid \overline{R_{2}\left(\Pi_{B}\right)}}\right)^{-1} \Pi_{B}\left(I-z \Pi_{B}\right)^{-1} g \\
& =\left(z \Pi_{B \mid \overline{R_{2}\left(\Pi_{B}\right)}}\right)^{-1}\left(\left(I-z \Pi_{B}\right)^{-1}-l\right) g \text { for all } g \in R_{2}\left(\Pi_{B}\right),
\end{aligned}
$$


which is true thanks to the relation: $\Pi_{B}\left(l-z \Pi_{B}\right)^{-1} g \in R_{2}\left(\Pi_{B}\right)$ for all $z \in R_{2}\left(\Pi_{B}\right)$. The boundedness of $\Pi_{B}{ }^{-1}$ from $R_{2}\left(\Pi_{B}\right)$ with $L_{2}$-metric into $L_{q}$ implies (b) in a similar way:

$$
R_{2}\left(\Pi_{B}\right) \stackrel{z^{-1}\left(I-R\left(z \Pi_{B}\right)\right)}{\longrightarrow} R_{2}\left(\Pi_{B}\right) \stackrel{\Pi_{B}^{-1}}{\longrightarrow} L_{q}
$$

The next two Lemmas and two Corollaries follow from interpolation theory. In particular, Lemma 4.8 is a consequence of Lemma 4.9 and is a generalization of results from $[5,15]$.

Lemma 4.8. Assume $p_{0}, p_{1}, q_{0}, q_{1}, \eta \in[1, \infty), p_{0} \leq q_{0}, p_{1} \leq q_{1}, \theta \in(0,1)$,

$$
\begin{gathered}
p_{\theta}^{-1}=(1-\theta) p_{0}^{-1}+\theta p_{1}^{-1}, \quad q_{\theta}^{-1}=(1-\theta) q_{0}^{-1}+\theta q_{1}^{-1}, \\
\left\{R\left(z e^{ \pm i \prime} \cdot\right)\right\}_{z \in S_{0}^{d}} \in \bar{D}\left(A, S_{0}^{d}, p_{0} . q_{0}, X_{0}\right) \cap \bar{D}\left(A, S_{0}^{d}, p_{1}, q_{1}, N, \dot{X}_{1}\right),
\end{gathered}
$$

and that $X_{0}, X_{1}$ are complemented subspaces of $L_{p_{0}}, L_{p_{1} . \rho_{t .1}^{N}}^{k}$ respectively, such that

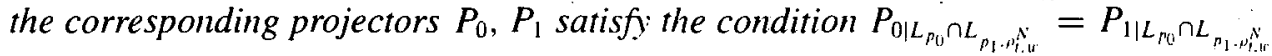
uniformly for $t>0$ and $w \in \mathbb{R}^{n}$. Then we have

$$
\left\{R\left(z e^{ \pm i \prime} \cdot\right)\right\}_{z \in S_{0}^{d}} \in \bar{D}\left(A, S_{0}, p_{\theta}, q_{\theta}, \theta N,\left[X_{0}, X_{1}\right]_{\theta, p^{\prime}}\right)
$$

PROOF. Utilising $\left(L_{p_{0}}, L_{p_{1}, \rho_{i, u}^{N}}\right)_{\theta, p_{\theta}}=L_{p_{t,}, \rho_{l, k}^{N u}}$ and $\left(L_{y 0} . L_{q_{t}}\right)_{\theta \cdot p_{\theta}}=L_{q_{t}, p_{\theta}} \subset L_{q_{\theta}}$, we apply Lemma 4.9 to the compatible pairs $\left(L_{p_{0}}, L_{p_{1}, \rho_{i . t}^{v}}\right)$ and $\left(X_{0}, X_{1}\right)$ in order to interpolate the family $\left\{R\left(z e^{ \pm i r^{\prime}} \cdot\right)\right\}_{z \in S_{0}^{d}}$.

Lemma 4.9. ([11,26]) For $p \in[1, \infty], \theta \in(0,1)$, let $\left(A_{0}, A_{1}\right)$. be a compatible couple of Banach spaces, and $B$ be a complemented subspace of $A_{0}+A_{2}$, whose projector $P \in \mathscr{L}\left(A_{0}\right) \cap \mathscr{L}\left(A_{1}\right)$. Then $\left(A_{0} \cap B, A_{1} \cap B\right)$ is also compatible and

$$
\left(A_{0} \cap B, A_{1} \cap B\right)_{t . p}=\left(A_{0}, A_{1}\right)_{\theta, p} \cap B .
$$

COROLlary 4.10. For $q \in\left(q_{0}, q_{1}\right) \subset[1, \infty]$, one has

(a) $L_{q}=N_{q}(A) \oplus \overline{R_{q}(A)}$, if $L_{q_{0}}=N_{q_{0}}(A) \oplus \overline{R_{q_{0}}(A)}, L_{q_{1}}=N_{q_{1}}(A) \oplus \overline{R_{q_{1}}(A)}$, and $P_{0 . p \mid N_{2}(A)}=P_{0,2 i N_{p}(A)}$;

(b) $\mathbf{P}(q), \mathbf{P}(2)$ and $\mathbf{K}(q)$ imply

$$
\begin{gathered}
\overline{R_{p}\left(\Pi_{B}\right)}=\left(\overline{R_{q}\left(\Pi_{B}\right)}, \overline{R_{2}\left(\Pi_{B}\right)}\right)_{\theta, p}, \quad \overline{R_{p}\left(\Gamma_{B}^{*}\right)}=\left(\overline{R_{q}\left(\Gamma_{B}^{*}\right)}, \overline{R_{2}\left(\Gamma_{B}^{*}\right)}\right)_{\theta, p} \\
\text { and } \overline{R_{p}(\Gamma)}=\left(\overline{R_{q}(\Gamma)}, \overline{R_{2}(\Gamma)}\right)_{\theta, p}
\end{gathered}
$$

for $1 / p=(1-\theta) / q+\dot{\theta} / 2$ 
(c) $\mathbf{P}^{*}(q), \mathbf{P}^{*}(2)$ and $\mathbf{K}^{*}(q)$ imply

$$
\begin{gathered}
\overline{R_{p^{\prime}}\left(\Pi_{B^{*}}^{*}\right)}=\left(\overline{R_{q^{\prime}}\left(\Pi_{B^{*}}^{*}\right)}, \overline{R_{2}\left(\Pi_{B^{*}}^{*}\right)}\right)_{\theta, p^{\prime}}, \quad \overline{R_{p^{\prime}}\left(\Gamma_{B^{*}}\right)}=\left(\overline{R_{q^{\prime}}\left(\Gamma_{B^{*}}\right)}, \overline{R_{2}\left(\Gamma_{B^{*}}\right)}\right)_{\theta, p^{\prime}} \\
\text { and } \overline{R_{p^{\prime}}\left(\Gamma^{*}\right)}=\left(\overline{R_{q^{\prime}}\left(\Gamma^{*}\right)}, \overline{R_{2}\left(\Gamma^{*}\right)}\right)_{\theta, p^{\prime}}
\end{gathered}
$$

for $1 / p=(1-\theta) / q+\theta / 2$

PROOF. Part (a) is an immediate consequence of Lemma 4.9, while in the rest of the lemma, we use the part of Corollary 2.8 involving $\Pi_{B}^{-1} \Gamma, \Pi_{B}^{*-1} \Gamma^{*}$ and continuity considerations before the application of Lemma 4.9.

COROLlary 4.11. Assuming $r \in(p, q)$, one has:

(a) $q=2, \mathbf{P}(p), \mathbf{P}(q)$ and $\mathbf{K}(p)$ imply $\mathbf{P}(r)$, and $p=2, \mathbf{P}^{*}(p), \mathbf{P}^{*}(q)$ and $\mathbf{K}^{*}(q)$ imply $\mathbf{P}^{*}(r)$;

(b) $q=2, \mathbf{G}(p), \mathbf{G}(q)$ and $\mathbf{K}(p)$ imply $B_{i} \in \mathscr{L}\left(L_{r}\right), i=1,2$ and there exist bounded $\left(B_{1 \mid \overline{R_{r}\left(\Gamma^{*}\right)}}\right)^{-1},\left(B_{2 \mid \overline{R_{r}(\Gamma)}}\right)^{-1}$;

(c) $p=2, \mathbf{G}^{*}(p), \mathbf{G}^{*}(q)$ and $\mathbf{K}^{*}(q)$ imply $B_{i} \in \mathscr{L}\left(L_{r}\right), i=1,2$ and there exist bounded $\left(B_{1 \mid \overline{R_{r^{\prime}}\left(\Gamma^{*}\right)}}^{*}\right)^{-1},\left(B_{2 \mid \overline{R_{r^{\prime}}(\Gamma)}}^{*}\right)^{-1}$.

The next theorem was proved in [10] with slightly different notation.

THEOREM 4.12. (a) Assuming $\mathbf{G}(2)$, and that $\Pi_{B}$ is local and of type $\omega$ in $L_{2}$ for some $0 \leq \omega<v<\pi / 2$, one has $\left\{R\left(z e^{ \pm i \nu} \Pi_{B}\right)\right\}_{z \in S_{0}^{d}} \subset \mathscr{L}\left(L_{2}\right)$ and $\left\{R\left(z e^{ \pm i i^{\prime}} \cdot\right)\right\}_{z \in S_{0}^{\prime}} \in$ $\bar{D}\left(\Pi_{B}, S_{0}, 2,2, N\right)$ for any $N>0$.

(b) Assuming $\mathbf{G}(2)$, and that $\Pi_{B^{*}}^{*}$ is local and of type $\omega$ in $L_{2}$ for some $0 \leq \omega<v<$ $\pi / 2$, one has $\left\{R\left(z e^{ \pm i \nu} \Pi_{B^{*}}^{*}\right)\right\}_{z \in S_{0}^{d}} \subset \mathscr{L}\left(L_{2}\right)$ and $\left\{R\left(z e^{ \pm i v} \cdot\right)\right\}_{z \in S_{0}} \in \bar{D}\left(\Pi_{B^{*}}^{*}, S_{0}^{d}, 2,2, N\right)$ for any $N>0$.

REMARK 6. It was proved in [10] that if $B_{1}$ is $\omega_{1}$-accretive on $R_{2}\left(\Gamma^{*}\right)$ and $B_{2}$ is $\omega_{2}$-accretive on $R_{2}(\Gamma)$ for some $\omega_{1}, \omega_{2} \in[0, \pi / 2)$, then $\Pi_{B}$ and $\Pi_{B^{\star}}^{*}$ are of type $\omega=\left(\omega_{1}+\omega_{2}\right) / 2$.

The next lemma is an immediate consequence of definitions.

LEMMA 4.13. For $p, q \in[1, \infty]$, an operator $A$ and a subspace $Y \subset L_{p}$, assume that $L_{p}=N_{p}(A) \oplus Y$, and $Y$ possesses the localization property. Then, for every subset $\left\{f_{z}\right\} \in \bar{H}_{\infty}^{0}\left(S_{\mu}^{d} \cup\{0\}\right)$, we have

$$
\left\{f_{z}\right\} \in \bar{D}(A, p, q, N, Y) \quad \text { if and only if } \quad\left\{f_{z}\right\} \in \bar{D}(A, p, q, N) \text {. }
$$


[25] Extrapolation of functional calculus, embedding theorems and Littlewood-Paley inequalities 321

THEOREM 4.14. (a) Assume $q \in(1,2), L_{q}=N_{q}\left(\Pi_{B}\right) \oplus \overline{R_{q}\left(\Pi_{B}\right)}$ and, uniformly by $t>0$ and $w \in \mathbb{R}^{n}, L_{2, \rho_{i, u}^{M}}=N_{2, \rho_{i, w}^{M}}\left(\Pi_{B}\right) \oplus \overline{R_{2, \rho_{i, w}^{M}}\left(\Pi_{B}\right)}, P_{0, q \mid N_{2 . \rho_{i, w}^{M}}\left(\Pi_{B}\right)}=P_{0,2 \mid N_{q}\left(\Pi_{B}\right)}$, $\left\{R\left(z e^{ \pm i \nu} \cdot\right)\right\}_{z \in S_{0}^{d}} \subset \bar{D}\left(\Pi_{B}, S_{0}^{d}, 2,2, M, \overline{R_{2}\left(\Pi_{B}\right)}\right)$ for any $M>0$, and $\mathbf{S}\left(\Pi_{B}, q, 2, X\right)$, where $\Pi_{B}(X)$ is $L_{q}$-dense in $\overline{R_{q}\left(\Pi_{B}\right)}$. Let, in addition, the set $\left\{R\left( \pm e^{ \pm i v} \Pi_{B}\right)\right\}$ be in $\mathscr{L}\left(L_{2}\right)$ for some $0 \leq \omega<v<\pi / 2$. Assume also the homogeneity property $\mathbf{H}$ with respect to all these conditions. Then, for any $p \in(q, 2)$ and $N>0$, one has $\left\{R\left(z e^{ \pm i v} \cdot\right)\right\}_{z \in S_{0}^{d}} \in \bar{D}\left(\Pi_{B}, S_{0}^{d}, p, 2, \overline{R_{p}\left(\Pi_{B}\right)}\right) \cap \bar{D}\left(\Pi_{B}, S_{0}^{d}, p, 2, N, \overline{R_{p}\left(\Pi_{B}\right)}\right)$.

(b) Assume $q \in(2, \infty),\left\{R\left(z e^{ \pm i v} \cdot\right)\right\}_{z \in S_{0}^{d}} \subset \bar{D}\left(\Pi_{B}, S_{0}^{d}, 2,2, M, \overline{R_{2}\left(\Pi_{B}\right)}\right)$ for any $M>0$, and $\mathbf{S}\left(\Pi_{B}, 2, q, X\right)$, where $\Pi_{B}(X)$ is $L_{2}$-dense in $\overline{R_{2}\left(\Pi_{B}\right)}$. Let, in addition, the set $\left\{R\left( \pm e^{ \pm i n} \Pi_{B}\right)\right\}$ be in $\mathscr{L}\left(L_{2}\right)$ for some $0 \leq \omega<\nu<\pi / 2$. Assume also the homogeneity property $\mathbf{H}$ with respect to all these conditions. Then one has $\left\{R\left(z e^{ \pm i \nu} \cdot\right)\right\}_{: \in S_{0}^{d}} \in \bar{D}\left(\Pi_{B}, S_{0}^{d}, 2, p, \overline{R_{2}\left(\Pi_{B}\right)}\right) \cap \bar{D}\left(\Pi_{B}, S_{0}^{d}, 2, p, N, \overline{R_{2}\left(\Pi_{B}\right)}\right)$ for any $p \in(2, q)$ and $N>0$.

PROOF. Because the proof of part (b) requires us to apply Lemma 4.9, we consider the proof of (a) only. Inclusion $\left\{R\left(z e^{ \pm i v} \cdot\right)\right\}_{z \in S_{0}^{d}} \in \bar{D}\left(\Pi_{B}, S_{0}, p, 2, \overline{R_{p}\left(\Pi_{B}\right)}\right)$ follows immediately from Lemma 4.7(a) and Lemma 4.9 applied to $\left(L_{q}, L_{2, \rho_{i . u}^{M}}\right)$ and $\left(\overline{R_{q}\left(\Pi_{B}\right)}, \overline{R_{2, \rho_{i, t}^{M}}\left(\Pi_{B}\right)}\right)$. The same lemma, and the inclusion

$$
\left\{R\left(z e^{ \pm i \nu} \cdot\right)\right\}_{z \in S_{0}^{d}} \subset \bar{D}\left(\Pi_{B}, S_{0}^{d}, 2,2, M, R_{2}\left(\Pi_{B}\right)\right) \quad \text { for any } M>0
$$

provide $\left\{R\left(z e^{ \pm i v \cdot}\right)\right\}_{z \in S_{0}^{d}} \in \bar{D}\left(\Pi_{B}, S_{0}^{d}, p, 2, N, \overline{R_{2}\left(\Pi_{B}\right)}\right)$ thanks to Lemma 4.8 .

\section{Extrapolation of operators}

The next theorem extends celebrated results from [12].

THEOREM 5.1. For $l \in \mathbb{N}, b>1, \delta>0$ and $p \in(1,2)$, let operators $T$ and $\left\{A_{t}\right\}_{t \geq 0}, A_{0}=I$ satisfy $T \in \mathscr{L}\left(L_{2}\right)$ and, for every $t>0, w \in \mathbb{R}^{n}$ and $\phi \in L_{p}$ with $\operatorname{supp} \phi \subset Q_{r}(w)$ and $\|\phi\|_{p} \leq 1$,

$$
\begin{gathered}
\left\|\left\{\alpha_{k}(t, w)\right\}_{k \in \mathbb{N}_{0}} \mid l_{1}\right\| \leq C_{1}<\infty, \quad \text { where } \\
\alpha_{k}(t, w):=t^{n / p-n / 2} b^{k n}\left\|T \Delta^{\prime} A_{t} \phi \mid L_{2}\left(Q_{b^{k+1}+\delta}(w) \backslash Q_{b^{k} t \delta}(w)\right)\right\|, \text { and } \\
t^{n / p-n / 2}\left(\left\|A_{t} \phi\left|L_{2}\left(Q_{t \delta}(w)\right)\|+\|\left\{\beta_{k}(t, w)\right\}_{k \in \mathbb{N}_{0}}\right| l_{1}\right\|\right) \leq C_{2}<\infty, \quad \text { where } \\
\beta_{k}(t, w):=b^{k n}\left\|A_{\imath} \phi \mid L_{2}\left(Q_{b^{k+1} 1_{1} \delta}(w) \backslash Q_{b^{k} \delta}(w)\right)\right\| .
\end{gathered}
$$

Then for all $u \in(p, 2)$ one has

$$
\max \left(\left\|T\left|\mathscr{L}\left(L_{p}, L_{p, \infty}\right)\|,\| T\right| \mathscr{L}\left(L_{u}, L_{u}\right)\right\|\right) \leq C\left(C_{1}+\left(1+C_{2}\left\|T \mid \mathscr{L}\left(L_{2}\right)\right\|\right)\right) .
$$


PROOF. Without loss of generality, one can assume that $\delta>2 n^{1 / 2} l$. Thus, we see that Theorem 5.1 becomes a particular case of Theorem III.6.3 of [2] (see also [3]), by taking $A=B=\mathbb{C}, \theta_{0}=\theta_{1}=\theta_{2}=v_{1}=w_{1}=2, v_{0}=w_{0}=p_{0}=p_{1}=p$, $t_{1}=t_{2}=1, \lambda_{0}=\lambda_{1}=v_{0}=v_{1}=n, D_{0}=\emptyset$ and $G(r, w):=I-\Delta^{\prime} A_{r}$ for any $Q_{r}(w) \subset \mathbb{R}^{n}$ in the conditions of the latter theorem.

The next theorem is obtained in $[5,6]$.

THEOREM 5.2. For $l \in \mathbb{N}, b>1, \delta>0, p \in(2, \infty)$, let operators $T$ and $\left\{A_{t}\right\}_{t \geq 0}$, $A_{0}=I$ satisfy $T \in \mathscr{L}\left(L_{2}\right)$, and, for every $t>0, \phi \in L_{2}$ and $\psi \in R_{2}(T)$,

$$
\begin{aligned}
t^{-n / 2}\left\|\Delta^{l} A_{t} T \phi \mid L_{2}\left(Q_{t}(\cdot)\right)\right\| & \leq C_{1} M_{2} \phi \quad \text { and } \\
t^{-n / p}\left\|A_{t} \psi \mid L_{p}\left(Q_{t}(\cdot)\right)\right\| & \leq C_{2} M_{2} \psi .
\end{aligned}
$$

Then one has $\left\|T \mid \mathscr{L}\left(L_{u}, L_{u}\right)\right\| \leq C\left(C_{1}+\left(1+C_{2}\left\|T \mid \mathscr{L}\left(L_{2}\right)\right\|\right)\right)$ for all $u \in(2, p)$.

\section{Further generalizations}

In this section, in addition to Remark 2(a), we discuss two ways to generalize the results of Sections 3 and 4, which will be partially employed in Part II of the article, devoted to applications.

First, let us observe that, similarly to [14], one can substitute Lebesgue spaces $L_{p}, p \in(1, \infty)$ with their closed subspaces $Y_{p}$. However, in our case, we should require them to form an interpolation scale and support the duality pairing $Y_{p}^{*}=Y_{p^{\prime}}$. Thanks to Lemma 4.9, it is sufficient to assume the existence of a bounded projector $P$ on $L_{q_{\mathrm{v}}}+L_{q_{s}^{\prime}}$ such that $P\left(L_{p}\right)=Y_{p}$ and $\left(P_{\mid L_{p}}\right)^{*}=P_{1 L_{p^{\prime}}}$. It is especially useful in situations where some properties are not satisfied on the whole Lebesgue space $L_{p}$, but hold on $Y_{p}$. The simplest examples appear if one can choose $Y_{p}=\overline{R_{p}(\Gamma)}$, or $Y_{p}=\overline{R_{p}\left(\Gamma_{B}^{*}\right)}$ and so on. In these cases, we do not need, for instance, the Projection property.

The second line of the generalization shows a way to avoid problems related to the Calderón-Zygmund property achieved through the sufficient condition from Remark 4(a). Namely, if $\overline{R_{p}\left(\Pi_{B}\right)}$ does not possess it, then we may find a bigger subspace (subset) $L_{p} \supset Z_{p} \supset \overline{R_{p}\left(\Pi_{B}\right)}$ satisfying this sufficient condition and a bounded operator $B: Y_{p} \rightarrow \overline{R_{p}\left(\Pi_{B}\right)}$ satisfying $B_{\mid \overline{R_{p}\left(\Pi_{B}\right)}}=I_{\mid \overline{R_{p}\left(\Pi_{B}\right)}}$. Indeed, in this case, it is sufficient to prove the boundedness of $f(A) B$ on $Z_{p}$ and consider the restriction $f(A) B_{\mid \overline{R_{p}\left(\Pi_{B}\right)}}$. A particular example of such a $B$ is a projector on $\overline{R_{p}\left(\Pi_{B}\right)}$. Such a generalization requires only one additional step: one needs to prove the offdiagonal-by-space estimates for the operator $B$. 
[27] Extrapolation of functional calculus, embedding theorems and Littlewood-Paley inequalities 323

\section{Approach based on semigroups}

In this section, we provide an example revealing a disadvantage of the approach based on the off-diagonal estimates for semigroups.

7.1. An example In this subsection, we shall study off-diagonal properties of an example of an operator $\Gamma$ suggested by McIntosh to the author as the simplest one-dimensional case:

$$
\Gamma:\left(\begin{array}{l}
f_{1} \\
f_{2}
\end{array}\right) \mapsto\left(\begin{array}{cc}
0 & \mathrm{D} \\
0 & 0
\end{array}\right)\left(\begin{array}{l}
f_{1} \\
f_{2}
\end{array}\right)=\left(\begin{array}{c}
f_{2}^{\prime} \\
0
\end{array}\right), \quad \Gamma^{*}=\left(\begin{array}{cc}
0 & 0 \\
-\mathrm{D} & 0
\end{array}\right)=\left(\begin{array}{cc}
0 & 0 \\
-f^{\prime} & 0
\end{array}\right)
$$

where $f(x)=\left(f_{1}(x), f_{2}(x)\right)$ is a function of one-dimensional variable $x \in \mathbb{R}$.

Let us assume that $f(x)$ is the restriction $f(x)=g(x+0 i)$ of an analytic function $g$ satisfying the Riemann formulae

$$
g(x+i y)=\frac{\operatorname{sign} y}{\pi i} \int_{-\infty}^{\infty} \frac{f(t) d t}{t-x-i y} \text { for all } x \in \mathbb{R}, y \in \mathbb{R} \backslash\{0\} .
$$

Representing $\Pi$ in the form

$$
\Pi=\mathrm{D} \otimes J, \quad \text { where } J=\left(\begin{array}{cc}
0 & 1 \\
-1 & 0
\end{array}\right)
$$

is a matrix imaginary unit of the $2 \times 2$ matrix representation of the complex numbers, we have, for all $h \in \mathbb{C}$,

$$
e^{h \Pi}=I \cos (h \Pi)+J \sin (h \Pi)=I \frac{e^{i h D}+e^{-i h D}}{2}+J \frac{e^{i h D}-e^{-i h D}}{2 i} .
$$

By assuming the convergence radius of the Taylor expansion of $g(z)$ at every $z$ with $\operatorname{Im} z=0$ is greater than $|h|$, one has $e^{h \mathrm{D}} g(z)=T_{i h} g(z)=g(z+h)$, that is, $e^{h \mathrm{D}}$ is a shift operator. Therefore, we see that

$$
\begin{aligned}
e^{h \Pi} & =\frac{T_{i h}+T_{-i h}}{2} I+\frac{T_{i h}-T_{-i h}}{2 i} J, \quad \text { or } \\
e^{h \Pi} f(x) & =I \frac{e^{i h D} g(x)+e^{-i h D} g(x)}{2}+J \frac{e^{i h D} g(x)-e^{-i h D} g(x)}{2 i} \\
& =I \frac{g(x+i h)+g(x-i h)}{2}+J \frac{g(x+i h)-g(x-i h)}{2 i} .
\end{aligned}
$$

The identity $J^{2}=-I$ and (7.5) show the presence of the semigroup property

$$
e^{h_{1} \Pi} e^{h_{2} \Pi}=e^{\left(h_{1}+h_{2}\right) \Pi} .
$$


Let us mention that, for $h \neq 0$, formulae (7.2) and (7.6) imply

$$
e^{y \cdot \Pi} f(x)=\left(I f * \phi_{h}(x)-J f * \psi_{h}\right)
$$

$$
\text { where } \phi_{h}=\frac{1}{h} \phi\left(\frac{\dot{h}}{h}\right), \quad \psi_{h}=\frac{1}{h} \psi\left(\frac{\cdot}{h}\right) \text {, }
$$

and $\phi(x):=\left(1+x^{2}\right)^{-1} / \pi, \psi(x):=x \phi(x)$. Since $\phi \in L_{1}(\mathbb{R})$ and the convolution with $\psi$ is a Calderón-Zygmund operator, the semigroup $e^{i \Pi}=e^{\operatorname{Re}: \Pi} e^{i \operatorname{lm}: \Pi}$ is welldefined on $L_{p}(\mathbb{R})$ for all $p \in(1, \infty)$ and $\left\|e^{-\Pi} \mid \mathscr{L}\left(L_{p}\right)\right\| \leq C(p)$ for any $p \in(1, \infty)$. The semigroup property of $e^{i \Pi}$ is closely related to the identities

$$
\phi_{h+w}=\phi_{h} * \phi_{w}-\psi_{h} * \psi_{w}, \quad \psi_{h+w}=\phi_{h} * \psi_{w}+\psi_{h} * \phi_{w}, \quad h, w \in \mathbb{C} \backslash\{0\}
$$

reflecting, in turn,

$$
\varrho_{h+u}=\varrho_{h} * \varrho_{u} \quad \text { for } \varrho(x):=\phi(x)-i \psi(x)=\frac{1}{\pi}(1+i x)^{-1} .
$$

At the same time, we have that, for $z=-i y, y \in \mathbb{R}$,

$$
e^{-i y \Pi} f(x)=l \frac{f(x+y)+f(x-y)}{2}+J \frac{f(x+y)-f(x-y)}{2 i} .
$$

Thus. one cannot achieve off-diagonal estimates for this semigroup with an arbitrary $N$. unless $z$ is purely imaginary. In general. only $N=1$ implied by the convolution with $\psi$ can be gained.

\section{Acknowledgements}

The author would like to thank A. McIntosh for his excellent introduction to the circle of questions related to the Kato Square root problem and the $L_{2}$-theory presented in [10] and for his suggestion to use the resolvent approach.

The author also takes this opportunity to express his gratitude to the Mathematical Sciences Institute of the Australian National University, where this work has been undertaken.

\section{References}

[1] S. S. Ajiev. 'Anisotropic supersingular integral operators and approximation formula', to appear in Math. Inequal. Appl.

[2] - Singular and supersingular operators on function spaces, approximation and extrapolation (Ph.D. Thesis. Australian National University: Canberra. 2003). 
[3] — ' On the boundedness of singular integral operators from some classes II'. Analysis Mathematica 32 (2006), 81-112.

[4] D. Albrecht, P. Auscher, X. Duong and A. Mcintosh, 'Operator theory and harmonic analysis', in: Instructional workshop on Analysis and Geometry; Part III (Canberra, 1995), volume 34 of Proc. Centre Math. Appl. Austral. Nat. Univ. (1996) pp. 77-136.

[5] P. Auscher. 'On $L^{p}$ estimates for square roots of second order elliptic operators on $\mathbb{R}^{n}$ ', Publ. Mat. 48 (2004), 159-186.

[6] P. Auscher. T. Coulhon. X. T. Duong and S. Hofmann, 'Riesz transform on manifolds and heat kernel regularity', Ann. Sci École. Norm Sup (4) 37 (2004), 911-957.

[7] P. Auscher, S. Hofmann, M. Lacey, A. McIntosh and Ph. Tchamitchian, 'The solution of Kato square root problem for second order elliptic operators on $\mathbb{R}^{n}$ '. Ann. of Math. (2) 156 (2002). 633-654.

[8] P. Auscher. S. Hofmann, A. McIntosh and Ph. Tchamitchian. 'The Kato square root problem for high order elliptic operators and systems on $\mathbb{R}^{n}$. J. Evol. Equ. 1 (2002), 361-385.

[9] P. Auscher and $\mathrm{Ph}$. Tchamitchian, 'Square root problem for divergence operators and related topics', Astérisque (1998), viiit+172.

[10] A. Axelsson. S. Keith and A. McIntosh. 'Functional calculus of Dirac operators with measurable coefficients', Invent. Math. 163 (2006), 455-497.

[11] M. S. Baouendi and G. Goulaouic, 'Commutation de l'intersection et des founcteurs d'interpolation', C. R. Acad. Sci. Paris Sér A-B 265 (1967), 313-315.

[12] S. Blunck and P. Kunstmann, 'Calderón-Zygmund theory for non-integral operators and the $H^{x}$. functional calculus', Rev. Mat. Iberoamericana 19 (2003), 919-942.

[13] R. Coifman, A. McIntosh and Y. Meyer. 'L'intégrale de Cauchy définit un opérateur borné sur $L_{2}\left(\mathbb{R}^{\prime \prime}\right)$ pour les courbes lipschitziennes'. Ann. of Math. (2) 116 (1982), 36 l-387.

[14] M. Cowling, I. Doust. A. McIntosh and A. Yagi, 'Banach space operators with a bounded $H^{x}$. calculus'. J. Aust. Math. Soc. 60 (1996), 51-89.

[15] E. B. Davies, 'Uniformly elliptic operators with measurable coefficients'. J. Funct. Anal. 132 (1995), 141-169.

[16] X. T. Duong and A. McIntosh. 'Singular integral operators with non-smooth kernels on irregular domains', Rev. Mat. Iberoamericana 15 (1999). 233-265.

[17] X.T. Duong and D. W. Robinson, 'Semigroup kernels, poisson bounds, and holomorphic functional calculus', J. Funct. Anal. 142 (1996). 89-128.

[18] W. Hebisch. 'A multiplier theorem for Schrödinger operators', Colloq. Math. 60-61 (1990), 659664.

[19] S. Hofmann and J. M. Martell, ' $L_{p}$-bounds for Reiesz transforms and square roots associated to second order elliptic operators'. Publ. Mat. 47 (2003). 497-515.

[20] S. Hofmann and A. McIntosh, 'The solution of the Kato problem in two dimensions', in: Proceedings of the conference on harmonic analysis and PDE (El Escorial, 2000), volume extra (2002) pp. 143-160.

[21] J. L. Lions, Equations différentielles operationelles et problèmes aux limites (Springer-Verlag, Berlin, 1961).

[22] J. M. Martell, 'Sharp maximal functions associated with approximations of the identity in spaces of homogeneous type and applications'. Studia Math 161 (2004), 113-145.

[23] A. McIntosh, 'On representing closed accretive sesquilinear forms as $\left(a^{1 / 2} u, a^{* 1 / 2} v\right)$ ', in: Nonlinear partial differential equations and their applications. College de France Seminar, Vol. III (Paris, 1980/1981), Res. Notes in Math. 70 (1982) pp. 252-267.

[24] - 'Operators which have an $H_{x}$ functional calculus', in: Miniconference on operator theory and partial differential equations (North Ryde, 1986), Proc. Centre Math. Anal. Austral. Nat. Univ. 14 (1986) pp. 210-231. 
[25] K. Moszyński, 'Concerning some version of the Lax-Milgram Lemma in normed spaces', Studia Math. LXVII (1980), 65-78.

[26] H. Triebel, Interpolation Theory, Function Spaces, Differential Operators (North-Holland Publishing Company, Amsterdam-Oxford, 1995).

School of Mathematics and Statistics

UNSW

Sydney NSW 2052

Australia

e-mail: ajievss@unsw.edu.au 